\title{
Numerical simulation of supernova-relevant laser-driven hydro experiments on OMEGA
}

\author{
A. R. Miles ${ }^{\text {a) }}$ \\ Lawrence Livermore National Laboratory, Livermore, California 94550 \\ and University of Maryland, College Park, Maryland 20741 \\ D. G. Braun, M. J. Edwards, and H. F. Robey \\ Lawrence Livermore National Laboratory, Livermore, California 94550 \\ R. P. Drake and D. R. Leibrandt \\ University of Michigan, Ann Arbor, Michigan 48109
}

(Received 29 May 2003; accepted 29 March 2004; published online 17 June 2004)

In ongoing experiments performed on the OMEGA laser [J. M. Soures et al., Phys. Plasmas 5, 2108 (1996)] at the University of Rochester Laboratory for Laser Energetics, nanosecond laser pulses are used to drive strong blast waves into two-layer targets. Perturbations on the interface between the two materials are unstable to the Richtmyer-Meshkov instability as a result of shock transit and the Rayleigh-Taylor instability during the deceleration-phase behind the shock front. These experiments are designed to produce a strongly shocked interface whose evolution is a scaled version of the unstable hydrogen-helium interface in core-collapse supernovae such as SN 1987A. The ultimate goal of this research is to develop an understanding of the effect of hydrodynamic instabilities and the resulting transition to turbulence on supernovae observables that remain as yet unexplained. The authors are, at present, particularly interested in the development of the RayleighTaylor instability through the late nonlinear stage, the transition to turbulence, and the subsequent transport of material within the turbulent region. In this paper, the results of numerical simulations of two-dimensional (2D) single and multimode experiments are presented. These simulations are run using the 2D Arbitrary Lagrangian Eulerian radiation hydrodynamics code CALE [R. T. Barton, Numerical Astrophysics (Jones and Bartlett, Boston, 1985)]. The simulation results are shown to compare well with experimental radiography. A buoyancy-drag model captures the behavior of the single-mode interface, but gives only partial agreement in the multimode cases. The RichtmyerMeshkov and target decompression contributions to the perturbation growth are both estimated and shown to be significant. Significant dependence of the simulation results on the material equation of state is demonstrated, and the prospect of continuing the experiments to conclusively demonstrate the transition to turbulence is discussed. (C) 2004 American Institute of Physics. [DOI: $10.1063 / 1.1753274]$

\section{INTRODUCTION}

The basic Rayleigh-Taylor (RT) instability criterion, ${ }^{1,2}$ neglecting certain potentially stabilizing factors such as surface tension, ${ }^{3}$ is the existence of antiparallel components of pressure and density gradients $(\nabla P \cdot \nabla \rho<0)$. When this condition is met at an interface between two materials, perturbations on the interface will grow in time. In the inviscid limit, the instability develops exponentially while the perturbations remain small (during the linear phase) with a growth rate given by ${ }^{4}$

$$
\gamma=\sqrt{\frac{k g A}{1+k L}},
$$

where $k$ is the perturbation wave number, $g$ is the acceleration, $A$ is the Atwood number, and $L$ is the density gradient scale length at the interface. At later times, initially sinusoidal perturbations grow into spikes of heavier fluid "fall-

${ }^{a)}$ Electronic mail: miles15@1lnl.gov ing" into lighter fluid and bubbles of lighter fluid "rising" into heavier fluid. For $A=1$, the bubbles rise with constant (terminal) velocity while spikes fall with constant acceleration in the nonlinear regime. ${ }^{5,6}$ When $A<1$, the spike eventually also reaches terminal velocity. ${ }^{6}$

There are several important nongravitational systems that are also strongly affected by the RT instability. For example, the RT instability has played an important historical role in the ability to magnetically confine hot plasmas, ${ }^{7}$ where the plasma serves as the heavier fluid. The confining magnetic field plays the role of the lighter fluid supporting the plasma against pseudogravitational centrifugal forces.

The RT instability criterion can also be satisfied at a material interface through which a blast wave has been transmitted from a heavier to a lighter fluid. ${ }^{8}$ As is illustrated in Fig. 1, since the pressure behind a blast wave is always falling in time at any fixed point and in distance behind the shock front (at least in the self-similar regime ${ }^{9}$ ), an interface generally becomes RT unstable when it transmits a blast 


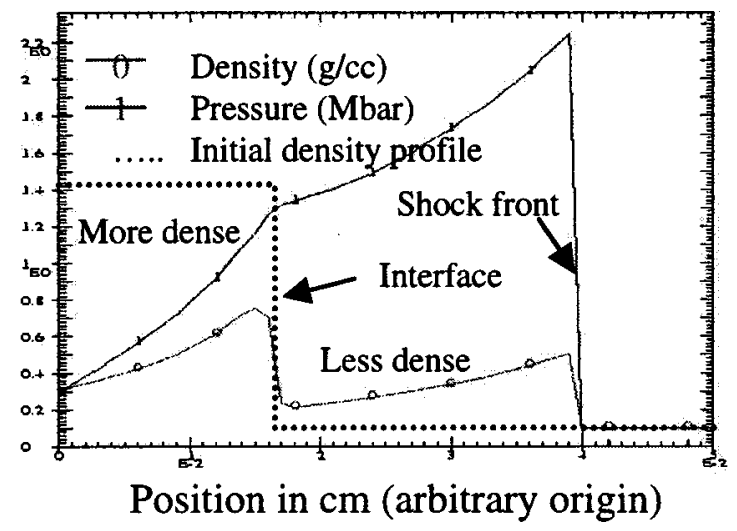

FIG. 1. Density (curve 0) and pressure (curve 1) behind a blast wave that has passed through a material interface from a $1.42 \mathrm{~g} / \mathrm{cc}$ plastic to a $0.1 \mathrm{~g} / \mathrm{cc}$ foam. The interface is RT unstable due to the presence of antiparallel density and pressure gradients at the interface. The dotted line shows the initial (pre-shock) density profile. The data are from a 1D CALE simulation of the experiment-relevant planar hydrodynamics at $14 \mathrm{~ns}$.

wave down a density gradient (i.e., from more dense to less dense material).

In inertial confinement fusion (ICF), pellets containing thermonuclear fuel are imploded by laser or particle beams or by x-rays generated by the interaction of such beams with the high- $Z$ walls of a hohlraum. ${ }^{4}$ The driving shock waves pass through the various material interfaces within the target, resulting in interface accelerations and decelerations that can in turn drive the RT instability. These processes tend to break up material shells and mix cold outer layers with the hot central region containing the fuel, and the resulting reduction in thermonuclear yield can be significant or even complete. Consequently, the RT instability has long been understood to be a major limiting factor in ICF.

Shock and blast waves are also common participants in astrophysical processes. ${ }^{10}$ In a core-collapse supernova, for example, the sudden release of an enormous amount of energy at the star's core drives a strong blast wave that propagates out through layers of progressively less dense matter. ${ }^{11,12}$ As the interfaces between these layers subsequently decelerate in the expansion fan behind the blast front, they are RT unstable. ${ }^{8}$ The potential significance of this phenomenon was realized with observations of SN1987A, when it was found that spherically symmetric explosion models failed to correctly predict the velocity and arrival time at the surface of heavy elements originating from the star's central regions. It has been suggested that the discrepancy results from the failure of the one-dimensional (1D) models to account for the turbulent (the Reynolds number has been estimated ${ }^{13}$ to be of order $10^{10}$ ) RT mixing that is certainly occurring at unstable interfaces. ${ }^{11,12,14,15}$

It has been suggested that, despite the huge difference in length and time scales, scaled experiments of some astrophysical systems can be fielded on high energy density (HED) facilities ${ }^{16,17}$ such as the OMEGA laser at the University of Rochester's Laboratory for Laser Energetics (LLE). ${ }^{18}$ This follows from the fact that, provided that viscosity as well as thermal and radiation transport can be neglected, the Euler equations are scale invariant. For example, consider two different systems (but with matching EOS) driven by strong shocks with velocities $v_{1}$ and $v_{2}$. If the post-shock density profiles are identical modulo a scale factor $h_{1} / h_{2}$, where $h_{1}$ and $h_{2}$ are characteristic length scales of the two systems, then the two systems will evolve identically on normalized time scales $h_{1} / v_{1}$ and $h_{2} / v_{2}$. The degree to which viscous effects are negligible is of course scale dependent, and they cannot be ignored in the vicinity of the Kolmogorov scale. Nevertheless, the hypothesis that scaled experiments can reproduce phenomenon simulated or observed on larger scales has been demonstrated for some laminar flows. ${ }^{15,19-21}$ Similarity in the structure of turbulent flows has also been observed across a wide range of scales for systems with similar characteristics, and appears to be quite general provided each system has sufficient time and available wave-number space for the development of a broad inertial range. ${ }^{22,23}$ The Reynolds number of the two flows need not be equal as long as it is in both cases sufficiently high so that the observable scales of interest are decoupled from the dissipative scales. Specifically, Dimotakis has shown that many flows exhibit a turbulent mixing transition at a critical Reynolds number of order $10^{4}$, above which their dependence on the Re is greatly diminished. $^{22}$

Such a scaling has been set out for the explosion phase of a core-collapse supernova, and experiments have been designed and conducted to begin to investigate several relevant issues in the laboratory. ${ }^{12,13,15,19}$ In this paper, we present the results of numerical simulations of a series of single-mode and multimode RT experiments carried out at the OMEGA facility. These are two-dimensional (2D) planar experiments for which the gross hydro is appropriately scaled to be relevant to core-collapse supernovae such as 1987A. For supernovae, characteristic length, pressure, and density scales are of order $10^{11} \mathrm{~cm}, 10^{-2} \mathrm{~g} / \mathrm{cc}$, and $10 \mathrm{Mbar}$, respectively. The characteristic time scale $h / \sqrt{ }(P / \rho)$ is thus of order $1000 \mathrm{~s}$. In the experiment, we have $h \sim 10^{2} \mathrm{um}, \rho \sim 1 \mathrm{~g} / \mathrm{cc}$, and $P$ $\sim 1 \mathrm{Mbar}$, which can be combined in the same way to give $\tau \sim 10 \mathrm{~ns}$. The $40 \mathrm{~ns}$ experiment therefore corresponds to the first several thousand seconds of the supernova's explosion phase.

In the experiment, the Reynolds number at the unstable interface grows up to be of order $10^{5}$. This is still far below the value of $10^{10}$ estimated for supernova flows, but significantly higher than Dimotakis' suggested sufficient condition for the turbulent mixing transition $\left(\operatorname{Re}_{\text {mix }} \sim 10^{4}\right) .{ }^{22}$ This suggests that the experiments are approaching a regime of true relevance to supernovae. During the course of the experiments, the evolving interface passes almost immediately through the linear phase of the RT instability and continues well into the deep nonlinear regime. The eventual goal of this line of experiments is to investigate this deep nonlinear phase, the eventual transition to turbulence, and especially the subsequent turbulent flow. This phase must be obtained for the development of an experimental test-bed that is truly representative of supernova hydrodynamics. In general, transition to turbulence is as yet poorly understood in compressible HED flows. ${ }^{10}$

We begin with brief descriptions of the experiment (more complete details of the experiments will be published 
separately) and the radiation-hydrodynamics code CALE, ${ }^{24}$ which is used for the simulations. We then present and discuss the simulation results, beginning with checks of the 1D hydrodynamics and proceeding on to single-, two-, and eight-mode perturbations. In each case, we demonstrate good agreement between the simulations and the experimental results. Our analysis shows that RM and target decompression effects must be considered in order to accurately describe the perturbation growth. A buoyancy-drag model is applied to the interface in each case. The model succeeds qualitatively in predicting the single-mode spike and bubble behavior. However, despite efforts to include the effects of bubble growth and merger in the application of the model to the multimode cases, it is there at best only partially successful. We also discuss the dependence of the simulated instability evolution on the laser energy, preheat, and the equation of state model, and show that the choice of EOS can significantly affect the growth rate and interface structure. Finally, we conclude with a summary of our results.

\section{EXPERIMENT}

In the experiments, ${ }^{23} 10$ of OMEGA's beams deliver a 1 ns pulse of $1 / 3 \mu \mathrm{m}$ laser light at one end of a directly driven cylindrical target (shown schematically in Fig. 2). The average laser intensity on the target is typically $6 \times 10^{14} \mathrm{~W} / \mathrm{cm}^{2}$. The laser energy is nominally $5 \mathrm{~kJ}$, but can vary by more than $1 \mathrm{~kJ}$ for experiments performed on different days.

The target consists of a $150 \mu \mathrm{m}$ long polyimide $\left(\mathrm{C}_{22} \mathrm{H}_{10} \mathrm{~N}_{2} \mathrm{O}_{4}\right)$ pusher/ablator section with a density of 1.41 $\mathrm{g} / \mathrm{cc}$. A single-mode or multimode $2 \mathrm{D}$ perturbation is machined into one end of the polyimide pusher, in which a 200 $\mu \mathrm{m}$ wide $4.3 \%$ brominated polystyrene $\left(\mathrm{C}_{500} \mathrm{H}_{457} \mathrm{Br}_{43}\right)$ radiographic tracer is embedded. The tracer serves to minimize edge and parallax effects during target imaging via side-on $\mathrm{x}$-ray radiography. With a density of $1.42 \mathrm{~g} / \mathrm{cc}$, the tracer is very nearly mass matched to the pusher so as to minimize any effects of the pusher-tracer boundary on the instability evolution. A $1.9 \mathrm{~mm}$ carbon foam payload [carbonized resorcinol formaldehyde (CRF) with density $0.1 \mathrm{~g} / \mathrm{cc}]$ is brought into contact with the pusher, and the resulting assembly rests within a cylindrical beryllium shock tube with an inner radius of $400 \mu \mathrm{m}$. Because the perturbation is machined into the plastic pusher but not into the foam payload, the two materials are actually in contact only at the highest peaks of the perturbation. As a result, there exists a gap between the two materials. Since target assembly takes place in air at atmospheric pressure, the gap is initially air filled. The extent to which that air subsequently diffuses out of the gap has not been quantified. The effect of $\mathrm{x}$-ray and electron preheat on the interface has also not been measured. X-ray preheat has been evaluated by simulations, however, as discussed below.

Phase plate beam smoothing results in a super-Gaussian spatial profile characterized by half-width $r_{0}=412 \mu \mathrm{m}$ and order $n=4.7$. This gives fairly uniform illumination on the target within the $400 \mu \mathrm{m}$ inner radius of the shock tube. As a result, the plastic-foam interface and the transmitted shock remain nearly planer throughout the experiment.

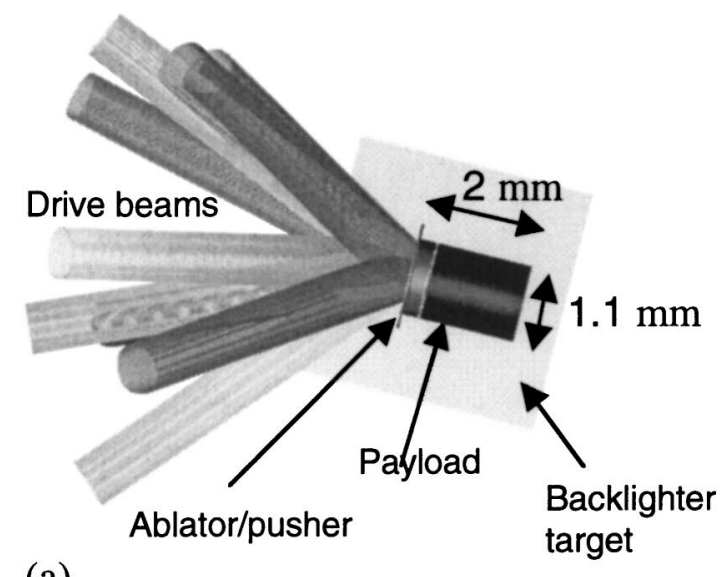

(a)

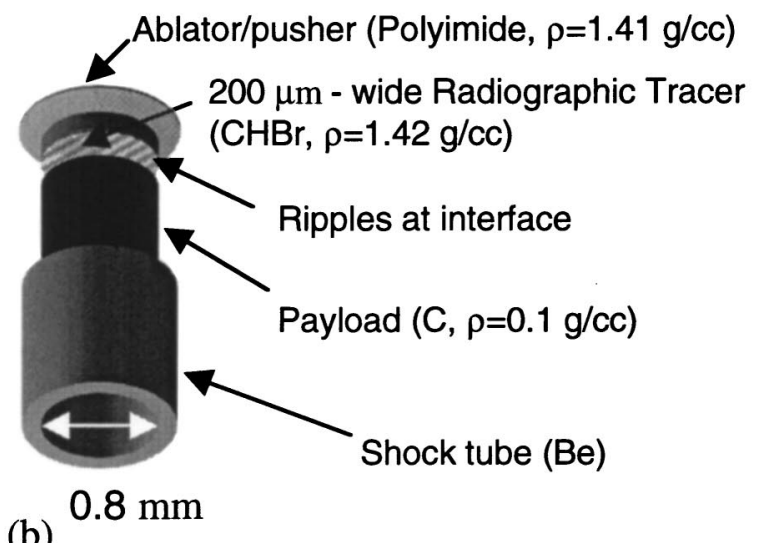

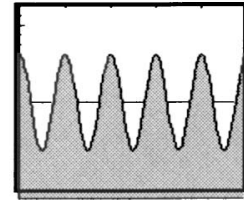

(c)

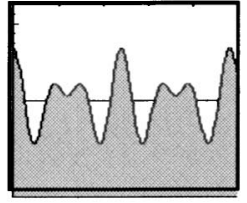

(d)

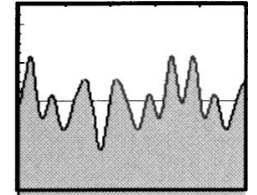

(e)
FIG. 2. Target schematic showing (a) overall experiment configuration, (b) an exploded view of the target package, and imposed interfacial perturbations for (c) single-mode, (d) two-mode, and (e) eight-mode experiments.

The experiments are diagnosed via side-on and face-on $\mathrm{x}$-ray radiography throughout their duration of up to $40 \mathrm{~ns}$. This is done with titanium back-lighters that produce $4.7 \mathrm{keV}$ photons for target imaging. The detector is a gatedmicrochannel-plate-intensified $\mathrm{x}$-ray camera with spatial resolution of about $10 \mu \mathrm{m}$, a gating time of about $250 \mathrm{ps,}$ and a quantum efficiency of $4 \%$. The field of view is set by the backlighter spot size of about $750 \mu \mathrm{m}$. The effect of the brominated tracer layer is illustrated by considering the $\mathrm{x}$-ray transmission through the various materials. Using the densities obtained at $10 \mathrm{~ns}$ from a 1D simulation, the side-on transmission through the foam side of the interface is 0.62 . Normalized to this value, the transmission through the plastic side of the interface is 0.021 . Without the tracer layer, it would be 0.18 . Thus, the contrast between the spike and bubble is nearly 9 times greater with the tracer layer than it would be without.

The incident laser pulse drives a strong shock wave (with Mach number $M \approx 15$ ) into the plastic pusher. When 
the laser pulse is terminated, the target begins to expand as a rarefaction wave is launched into the target. By the time the incident shock has reached the interface, the rarefaction wave has overtaken it, resulting in the formation of a blast wave. The blast wave crosses the interface at about $2.5 \mathrm{~ns}$, accelerating it to about $70 \mu \mathrm{m} / \mathrm{ns}$ and depositing vorticity that will subsequently drive Richtmyer-Meshkov ${ }^{25,26}$ (RM) growth. After passage of the blast wave, the interface begins to decelerate, and continues to do so throughout the remainder of the experiment. During the deceleration phase, the interface is RT unstable. In addition to the RM and RT instabilities, target decompression occurs during the experiment, and is responsible for about $50 \%$ of the total perturbation growth.

\section{SIMULATION}

We use CALE (for C-based Arbitrary Lagrangian Eulerian) to simulate the experiments. CALE is a $2 \mathrm{D}$ radiation hydrodynamics code that uses a finite-differencing method to numerically solve the Euler equations. ${ }^{24}$ As an ALE code, it mixes elements of Eulerian and Lagrangian techniques in order to inhibit mesh entanglement. Although we generally run these simulations in ALE mode, some Eulerian calculations were also performed for comparison. Unless otherwise stated, planar symmetry is specified in the direction perpendicular to the computational domain (i.e., $x-y$ Cartesian rather than axisymmetric), and electron conduction is included.

The initial length of the computational grid is typically 2 $\mathrm{mm}$. In the single-mode simulations, its width is generally 25 $\mu \mathrm{m}$, equal to one-half the perturbation wavelength. However, simulations were also carried out with full wavelengths and multiple wavelengths. In these cases, as in the half-mode simulations, reflection boundary conditions are specified on the boundaries parallel to the target's symmetry axis, while free boundary conditions are used on the orthogonal boundaries. Finally, full-target simulations that include the shock tube were performed to verify that the large-scale hydrodynamics do not adversely affect the experiment (see Sec. IV B).

Numerical radiographs can be produced by CALE and directly compared with experimental data. The numerical images, which depend on user-input x-ray opacities and the backlighter energy, provide an instantaneous snapshot of the system. Because the 250 ps gating time of the actual detector is short compared to the hydrodynamic time scales, the approximation of perfect temporal resolution is reasonable. The numerical radiographs do not account for the finite instrumental resolution or the statistical noise in the experimental radiography. We therefore always distinguish them from "simulated" x-ray radiographs, which can be made from the CALE results by folding in photon statistics and the pinhole resolution of the framing camera, thereby allowing for more realistic side-by-side comparisons with the data.

The pusher density in the simulations is $1.42 \mathrm{~g} / \mathrm{cc}-$ equal to that of the brominated plastic tracer layer, and the foam density is $100 \mathrm{mg} / \mathrm{cc}$, as in the experiment. The gap between plastic and foam is included in the simulations, and its density is typically set to $1 \mathrm{mg} / \mathrm{cc}$. This should be considered an upper bound on the actual gap density, but simulations run with lower values yield virtually identical results. Although it has been shown that such a gap can have a significant effect in laser-driven RM instability experiments on the spike and bubble structure as well as the perturbation amplitude, ${ }^{27}$ simulations suggest that in these RT experiments, which involve very strong shocks and initially smallamplitude perturbations, only the amplitude is significantly affected by the presence of an air-filled gap. Omission of the gap results in a decrease of up to $10 \%$ in the amplitude and amplitude growth rate. This difference is sufficient to warrant inclusion of the gap in the simulations.

In order to investigate the dependence of the instability evolution on the choice of equation of state (EOS) model, we have run simulations with tabular EOS as well as perfect gas with various choices of adiabatic indices for the plastic and foam. We use two commonly used sets of EOS tables, called LEOS (derived from QEOS ${ }^{28}$ ) and EOP (used, for example, in Ref. 29), which are both Thomas-Fermi-type. These models do not take into account the material structure, but we do not consider this a bad approximation given that the actual foam pore size is no more than about $0.1 \mu \mathrm{m}$. As will be shown in Sec. IV D, the EOS choice can nevertheless have a significant effect on the instability evolution.

The initial material temperatures in the CALE simulations discussed above are typically $25 \mathrm{meV}$ (290 K). After laser deposition, radiation effects and electron preheat are generally not included, so the interface (along with all the preshock target) remains cold until the arrival of the shock. Significant levels of preheat would cause the interface to move and its perturbation structure to change prior to shock transmission. If the plastic side of the interface was heated sufficiently to cause melt, the interface would begin to move away from the laser end of the target, possibly driving a shock into the foam, and the perturbation amplitude would decrease (remaining stable) and possibly become nonsinusoidal.

A 2D LASNEX ${ }^{30}$ calculation including radiation transport predicts that material at the interface is preheated to a temperature of about $0.4 \mathrm{eV}$ before the arrival of the shock. In order to estimate the effect this might have on the subsequent instability evolution, a simulation was run in which the initial temperature of all materials was increased to $0.4 \mathrm{eV}$ (see Fig. 3). The effect on the spike and bubble shape is relatively small, and the perturbation amplitude is reduced by about $10 \%$. At the time of shock arrival at the interface, the amplitude has been reduced from 2.5 to $1.9 \mu \mathrm{m}$, which certainly accounts for some of the subsequent growth reduction. At later times, the perturbation growth is also inhibited by the increased thermal pressure of the preheated foam. However, the LASNEX calculation predicts that the level of preheat is less than $0.2 \mathrm{eV}$ beyond about $50 \mu \mathrm{m}$ into the foam. Consequently, the resulting reduction could be considerably less than the $10 \%$ seen in the CALE calculation with unrealistically uniform $0.4 \mathrm{eV}$ preheat. Since the main effect of preheat is to reduce the initial amplitude, another simulation was run in which the initial temperature was only $25 \mathrm{meV}$ but the initial amplitude was set to $2.0 \mu \mathrm{m}$. The resulting 


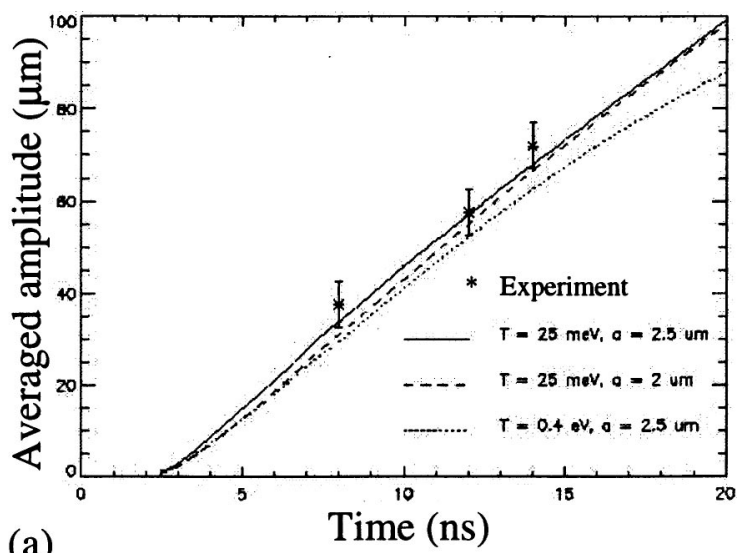

(a)

\section{Unshocked foam}

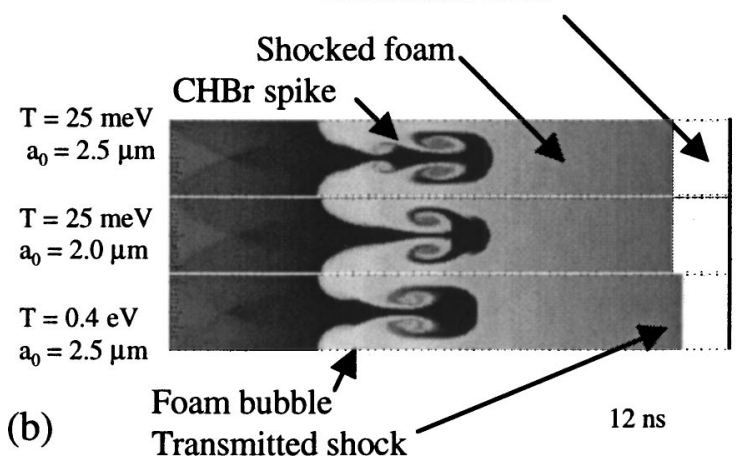

FIG. 3. Estimation of the effect of preheat. LASNEX predicts that the interface is preheated to $0.4 \mathrm{eV}$ before shock refraction. (a) A CALE simulation run with the initial temperature of all materials increased from $25 \mathrm{meV}$ to $0.4 \mathrm{eV}$ yields a decreased perturbation amplitude. The pre-shock amplitude is reduced from $2.5 \mu \mathrm{m}$ to $1.9 \mu \mathrm{m}$. A third calculation with a low initial temperature but with the initial amplitude reduced to $2.0 \mu \mathrm{m}$ does not differ significantly at later times from the larger initial amplitude case. The results suggest that the effect of preheat on the amplitude is less than $5 \%$ after shock refraction. (b) Numerical radiographs show that the effect on the shape of the spike and bubble is relatively small. All three simulations were run with EOP EOS tables for all materials. The shock is moving from left to right, the dark region on the left (including the spike) is the plastic pusher material, the lighter region to the right (including the bubble) is shocked foam, and the white region on the far right is unshocked foam.

amplitude reduction at later times was never greater than $5 \%$. Beyond $15 \mathrm{~ns}$, the amplitude histories are virtually identical. Consequently, we do not consider $\mathrm{x}$-ray preheat to have a significant impact on the instability evolution in these experiments.

Three different methods have been used in the simulations to drive the incident shock. In the first method, a timedependent velocity source is extracted from a 2D laserdriven LASNEX simulation and then input into CALE (see Fig. 4). This is usually done with the time-dependent pulse shape from an actual experiment, but we have found that a simple square pulse gives nearly identical results. In the first step of the second case, a 1D or 2D laser-driven LASNEX or CALE simulation is run. At the time that the laser turns off, the density, temperature, velocity, and position of the resulting shock-compressed slab is recorded. This information is then input into the full-sized 2D CALE simulation as a highpressure, high-velocity slab, which then evolves into a blast wave and subsequently drives the instability. The velocity

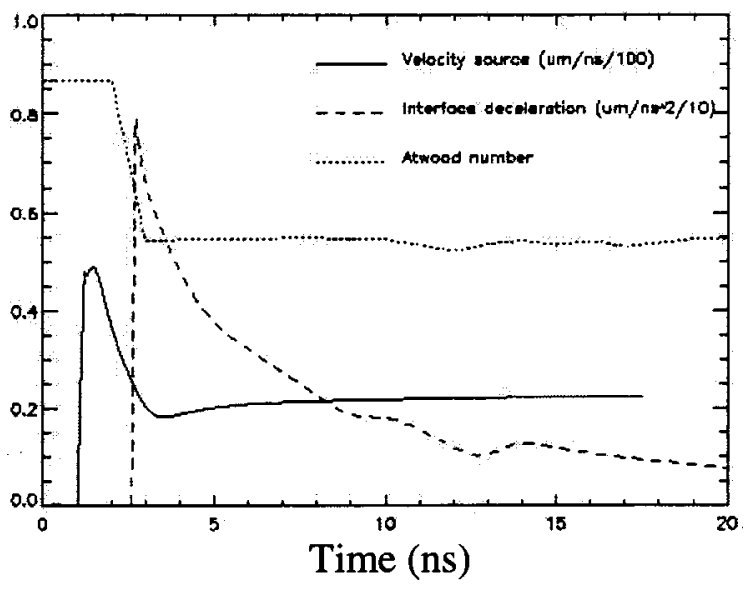

FIG. 4. CALE simulations are driven with a time-dependent velocity source extracted from a 2D LASNEX simulation which in turn is driven by a $3.5 \mathrm{~kJ}$ $1 \mathrm{~ns}$ laser pulse. The curve shown is the velocity of the on-axis fluid element initially located $70 \mu \mathrm{m}$ into the plastic pusher $(80 \mu \mathrm{m}$ from the plastic-foam interface). The plastic-foam interface deceleration and Atwood number from a 1D CALE simulation driven by this velocity source are also shown. The post-shock Atwood number remains nearly constant at about 0.54 .

drive is generally used in ALE calculations, while the slab drive is more naturally implemented in Eulerian runs. In the third method, CALE's laser beam package is used to directly drive the simulated target with a square pulse. The three methods yield virtually identical results (the agreement among the interface trajectories is at all times better than 2\%) when the energy deposition in the laser-driven case is spatially uniform. Since this is always the case in our halfwavelength simulations, we will in those cases not specify which mechanism was used. In full-target simulations, however, the laser drive has the advantage that it can be applied with the actual super-Gaussian intensity profile delivered by OMEGA, and so such cases will be presented with the drive mechanism used.

A resolution study was performed to ensure an adequate level of convergence in the simulations. In the study, the zero-time cell aspect ratio, defined as the ratio of the transverse to parallel cell dimensions, was held fixed at 5/3. Amplitude histories and numerical and simulated radiographs at 8 and 14 ns are shown in Fig. 5 from four simulations with transverse resolution ranging from 30 to 240 points per perturbation wavelength (ppw). The interface position (and therefore velocity) shows little variation from one simulation to another, and the shock position is nearly identical in all four cases. The variation in amplitude from the mean is in each case less than $6 \%$, and does appear to vary systematically with resolution. The most significant variation is in the small-scale details. Specifically, as the resolution increases, more and more Kelvin-Helmholtz $(\mathrm{KH})$ rollup appears along the interface, with an increasingly smaller minimum scale length. The variation of the late-time spike stalk on resolution is apparent in the simulated radiographs, and the calculation with the lowest resolution agrees best with the data. Though the increase in $\mathrm{KH}$ rollup with resolution has rather limited impact on experimentally observable scales, the question of its veracity should be considered in studies of the approach and transition to turbulence. Eulerian calcula- 


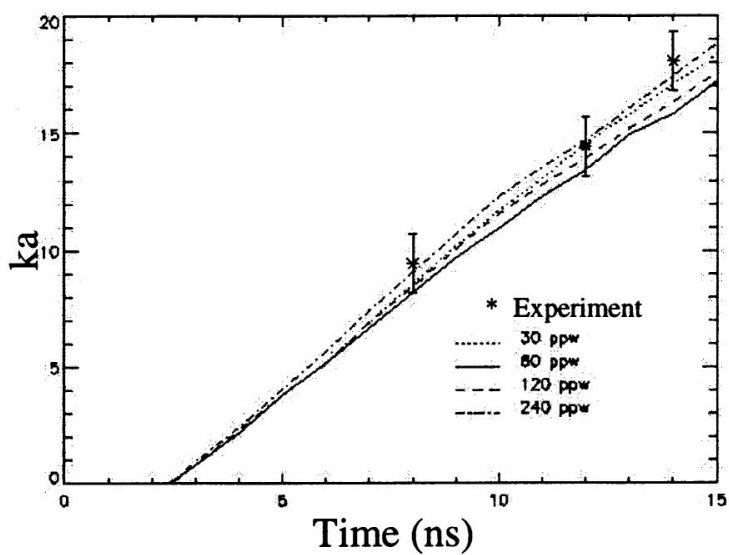

(a)
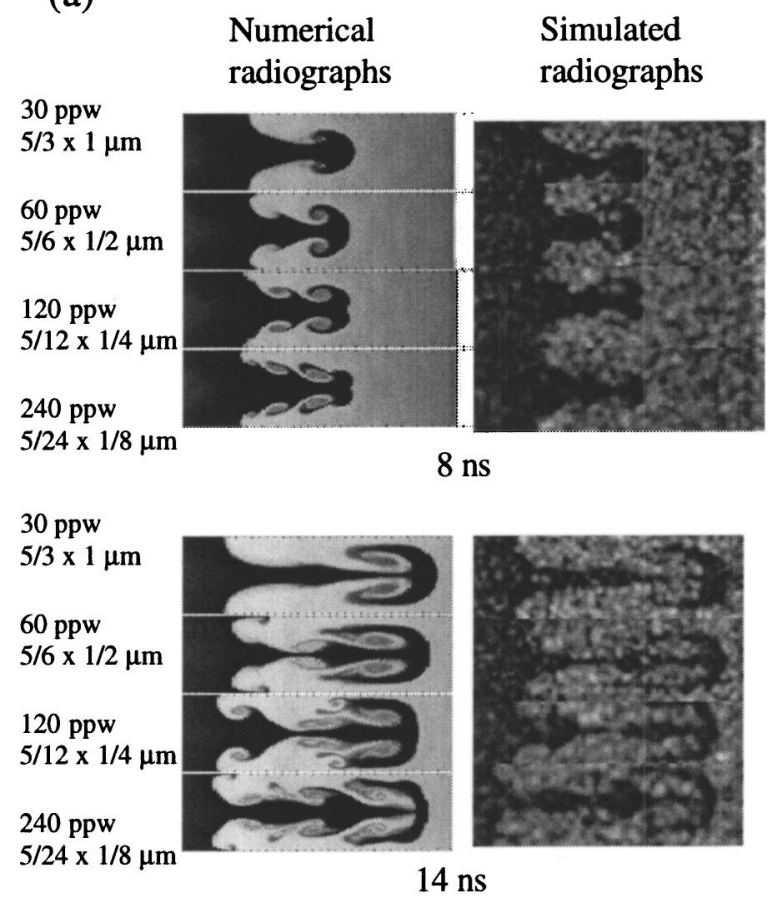

(b)

FIG. 5. Resolution study. (a) Amplitude histories and (b) numerical and simulated radiographs from CALE simulation at 8 and 14 ns. Simulated radiographs include the effects of the instrumental resolution and noise due to photon statistics. In each case, the cell dimension aspect ratio is $5 / 3$.

tions exhibit less small-scale KH activity than do ALE simulations run at the same transverse resolution (see Fig. 6). In most cases, the difference is too small to be resolved by the experiment. However, experimental radiography from the two-mode experiment at $13 \mathrm{~ns}$ appears to be better reproduced by the Eulerian calculation. Though not conclusive, this suggests that the relative increase in $\mathrm{KH}$ activity is perhaps stimulated by the grid motion algorithm selected in the ALE simulations.

Half-wavelength simulations are generally run with a transverse resolution of $60 \mathrm{ppw}$. This appears to be adequate to provide a sufficient level of convergence on the experimentally observable scales. The limited numerical resolution corresponds to a resulting limitation in Reynolds number. With 60 ppw, the simulation Reynolds number is about 1200

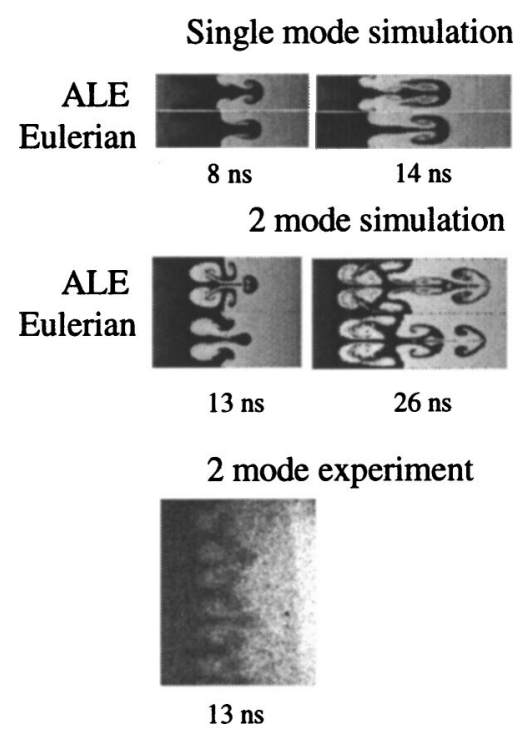

FIG. 6. ALE/Eulerian comparison for single-mode and two-mode targets. Kelvin-Helmholtz instability effects are more prominent when CALE is run in ALE mode than in Eulerian. In the two-mode case, the $T=13 \mathrm{~ns}$ result from the Eulerian calculation seems to agree better with the data, suggesting that these effects are exaggerated in the ALE runs.

when the value in the experiment is of order $10^{5}$. Thus, we do not expect the simulations to reproduce the smallest-scale features present in the experiment. Since these scales are also below the current experimental resolution, this limitation is important only if there is significant coupling between the large and small scales.

To summarize, a baseline calculation spans the entire target length and one-half of one perturbation wavelength in the transverse direction with resolution of $60 \mathrm{ppw}$. It includes an air-filled gap where the corrugated plastic interface comes into contact with the planar surface of the foam payload, and is run with planar symmetry in the direction perpendicular to the computational domain. The code's ALE feature is enabled, electron conduction is included, and the initial temperature is set to $25 \mathrm{meV}$ (no preheat). Finally, tabular EOS are used for all materials.

\section{SIMULATION RESULTS}

\section{A. Zero-order hydrodynamics}

A 1D simulation is used to investigate the experiment's zero-order hydrodynamics. When the EOP tables are used for all materials, the pressure and temperature in the pusher behind the incident shock are about $55 \mathrm{Mbar}$ and $25 \mathrm{eV}$, respectively, at the end of the $5 \mathrm{~kJ}, 1 \mathrm{~ns}$ laser pulse (45 Mbar and $20 \mathrm{eV}$ for $3.5 \mathrm{~kJ}$ pulse). Just before the shock reaches the interface, these values have fallen to about $40 \mathrm{Mbar}$ and 20 $\mathrm{eV}$ (or $25 \mathrm{Mbar}$ and $16 \mathrm{eV}$ with $3.5 \mathrm{~kJ}$ drive). Figure 7 shows the time dependence of the pressure at the interface, along with the density, temperature, and sound speed on both sides of the interface. The post-shock pressure at the interface is about 4.5 Mbar just after shock transmission and falls to about 0.5 Mbar by $40 \mathrm{~ns}$. Even at late times, the shock pressures are sufficiently high that foam crush is not an issue. 


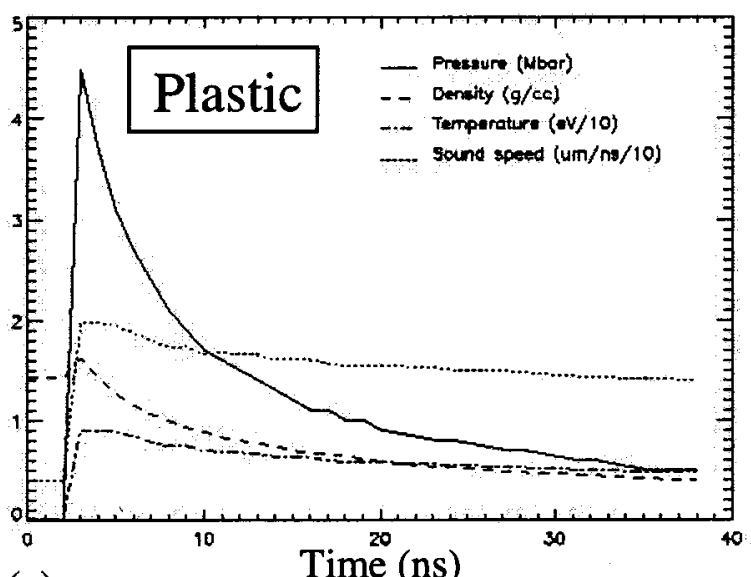

(a)

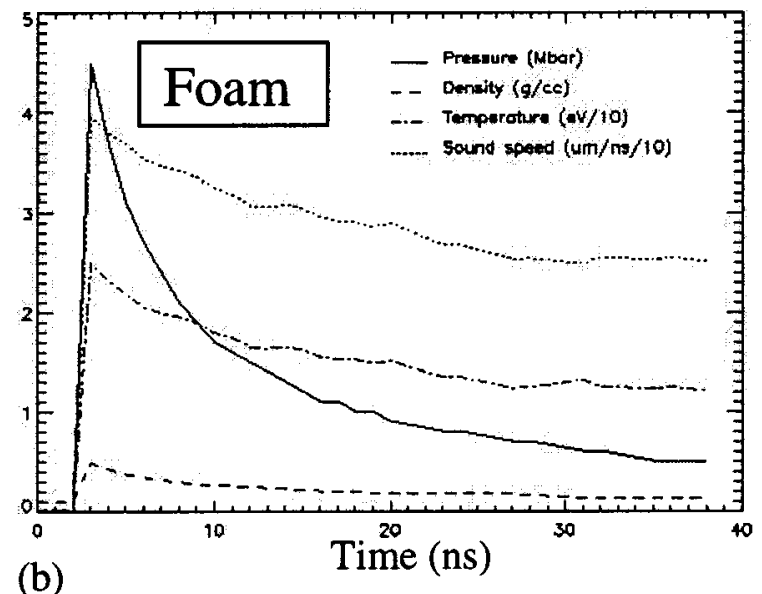

FIG. 7. Time dependence of fluid variables at the plastic-foam interface from a 1D CALE simulation: (a) Plastic side of the interface and (b) foam side of the interface. The pressure (solid line) and velocity (not shown) are continuous across the interface.

The pre-shock Atwood number $A$ is 0.87 , and the post-shock Atwood number $A^{*}$ is nearly constant at 0.54 (see Fig. 4).

The validity of the $1 \mathrm{D}$ hydrodynamics was verified by comparing the code's prediction of the shock and interface trajectories with experimental measurements, as shown in Fig. 8. The incident shock arrives at the interface rather quickly-in about $2.5 \mathrm{~ns}$, so the extent to which the comparison of the incident shock speed can be made is limited. Except in full-target simulations driven by CALE's laser package with the actual beam spatial profile, the transmitted shock speed is consistently too high for any reasonable EOS tried. The simulation results begin to deviate substantially from the data by approximately $15 \mathrm{~ns}$. The three late-time data points (one at $21 \mathrm{~ns}$ and two at $26 \mathrm{~ns}$ ) show that, after 20 ns, the simulated shock has advanced between 120 and 200 $\mu \mathrm{m}$ ahead of the actual shock position. This is between $9 \%$ and $14 \%$ of the distance traveled by the simulated shock at those times, and between $24 \%$ and $44 \%$ of the shockinterface separation. Correctly matching the shock-interface separation is important while the transmitted shock is close enough to the interface to significantly inhibit perturbation growth. This can happen either due to a shock-proximity effect, when the spike-shock distance is significantly less than the perturbation amplitude, or as a bounded-flow effect.
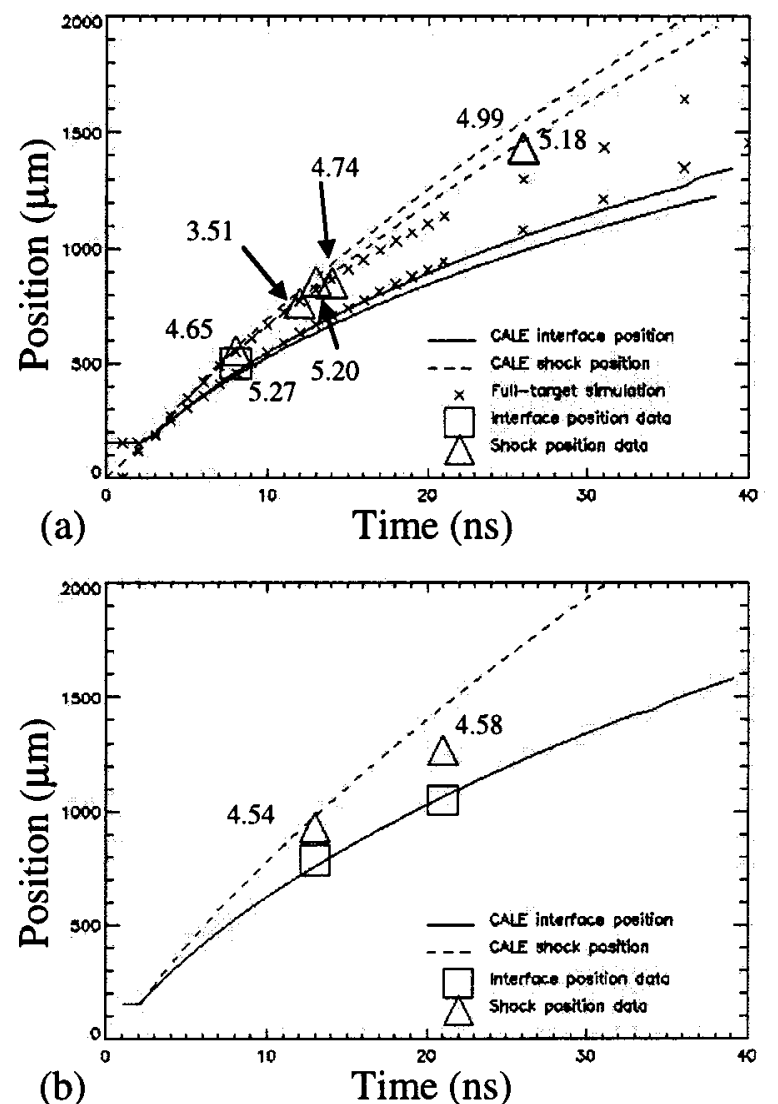

FIG. 8. Interface and shock trajectories with (a) $\rho_{\text {foam }}=100 \mathrm{mg} / \mathrm{cc}$ and (b) $\rho_{\text {foam }}=50 \mathrm{mg} / \mathrm{cc}$. In (a), results are included from simulations with both 5.0 $\mathrm{kJ}$ drive (upper curves) and $3.5 \mathrm{~kJ}$ drive (lower curves). In (b), the drive energy is $5 \mathrm{~kJ}$. The full-target simulation includes the Be shock tube and laser spot spatial profile as well as consequent edge effects including divergence and shock curvature. The plastic-foam interface is initially planar. All other CALE results are from 1D simulations. Experimental data are shown with points that are approximately four times the experimental error. The experimental energies (in $\mathrm{kJ}$ ) corresponding to each data point are included in the figures.

In our simulations, the ratio $\left(z_{\text {shock }}-z_{\text {spike }}\right) /(2 a)$, where $z_{\text {shock }}-z_{\text {spike }}$ is the distance from the spike tip to the transmitted shock and $2 a$ is the peak-to-valley amplitude, grows to 0.80 within $1 \mathrm{~ns}$ after shock refraction through the interface and subsequently climbs monotonically to about 1.20 . The experimental values of this ratio at 8,12 , and $14 \mathrm{~ns}$ are $0.91,0.71$, and 0.90 , respectively. Hecht et al. ${ }^{16}$ have considered the problem of a bubble rising into a fluid that is wallbounded from above. They find that the inhibiting effect of the wall on the bubble velocity decays exponentially with increasing bubble-wall separation as $e^{-2 k(d-z)}$, where $d$ and $z$ are the heights of the wall and bubble, respectively. If we assume for our case that the transmitted shock acts as a bounding wall and that spike growth suppression follows the same scaling as found for the bubble, then this effect becomes negligible when $z_{\text {shock }}-z_{\text {spike }} \gg 4 \mu \mathrm{m}$. This condition is satisfied within about $3 \mathrm{~ns}$ in the simulations, and probably in about $4 \mathrm{~ns}$ in the experiment. Consequently, the shock quickly moves far enough away that its effect on the perturbation growth is small except perhaps during the first few ns. The difference between measured and simulated shock speeds nevertheless does indicate that the simulations are 


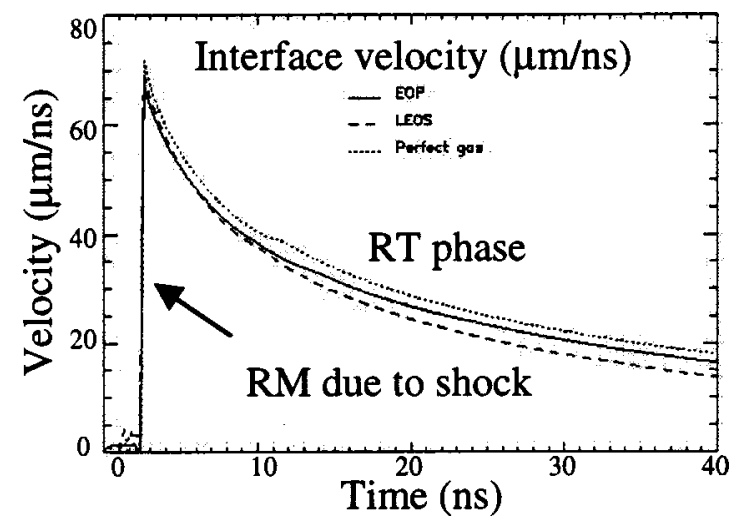

FIG. 9. Interface velocity from a 1D CALE simulation driven by a $3.5 \mathrm{~kJ} 1$ ns laser pulse. The shock induces RM growth, and the interface is RT unstable during subsequent deceleration phase.

missing something. We believe that that there are two contributing factors. First of all, the EOS tables may underpredict the foam compressibility in some regions of pressurevolume space. This point, along with its consequences on accurate modeling of the instability, will be further discussed in Sec. IV D. Second, the artificial planarity of the shock in the simulations also contributes to its anomalously high speed. Because of lateral expansion, the actual shock speed on the target axis is less than predicted by the 1D simulation. The full-target laser-driven simulation significantly overpredicts the shock curvature, and consequently actually underpredicts the shock speed at late times [see Fig. 8(a)]. We believe that this is due to imperfect modeling of the laser deposition. Since the shock appears nearly planar in the data, shock speed reduction due to lateral expansion is probably a lesser effect compared to the EOS.

The dependence of the zero-order hydrodynamics on the choice of EOS model used in CALE is shown in Fig. 9. In the simulations, the initial speed of the transmitted shock in the foam is about $70 \pm 3 \mu \mathrm{m} / \mathrm{ns}$, and the precise value in that range depends on both the drive energy and the EOS. The spread in results obtained with the various EOS models is comparable in magnitude to the variation caused by varying the drive energy over the range seen in the experiments.

\section{B. Single-mode perturbation}

The single-mode perturbation is characterized by a wavelength of $50 \mu \mathrm{m}$ and an amplitude of $2.5 \mu \mathrm{m}$. Side-on radiographs of the instability growth were obtained from 3 separate laser shots at 8, 12, and 14 ns [see Figs. 10(a)$10(\mathrm{c})]$. The laser energies corresponding to the data shown in Figs. $10(\mathrm{a})-10$ (c) are $4.65,3.50$, and $4.74 \mathrm{~kJ}$, respectively. The CALE-produced interface trajectories in Fig. 9 are from simulations driven by a $3.5 \mathrm{~kJ}$ pulse. When a $5 \mathrm{~kJ}$ pulse is used instead, the initial post-shock interface velocity is increased by about $4 \mu \mathrm{m} / \mathrm{ns}(6 \%)$, the displacement of the interface (at $40 \mathrm{~ns}$ ) from its initial position has also increased by $6 \%$ (from 1110 to $1180 \mu \mathrm{m}$ ), and the perturbation amplitude at $40 \mathrm{~ns}$ has increased by about $4 \%$. At the times of the three images in Fig. 10, the absolute amplitude increase is 3-5 $\mu \mathrm{m}$ (even less when the comparison is made at identical

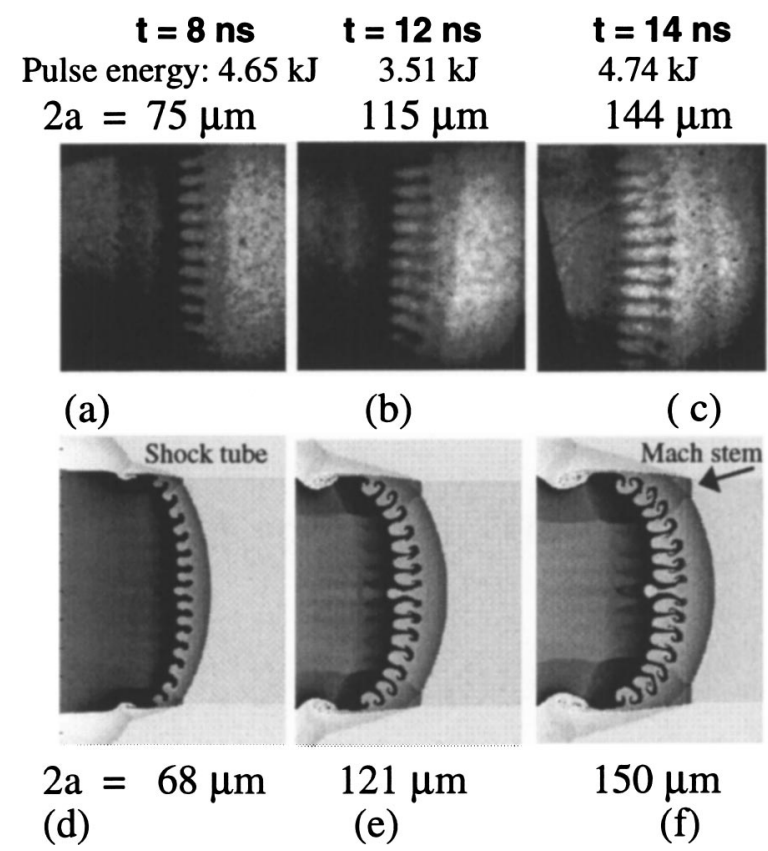

FIG. 10. (a)-(f) Single-mode data and simulation. The simulation was run in cylindrical coordinates (resulting in the unphysical interface asymmetry on axis) and driven by CALE's laser beam package with a super-Gaussian spatial profile characterized by half-width $r_{0}=412 \mu \mathrm{m}$ and order $n=4.7$. These parameters are as in the experiment.

interface position rather than time). Since this value is below the experimental resolution of about $10 \mu \mathrm{m}$, it is fair to conclude that, with present diagnostics, the existing uniformity of laser energy is satisfactory.

Numerical radiographs at the same times, all from one full-target simulation driven by a laser pulse with the correct super-Gaussian spatial profile, are shown in Figs. 10(d)10(f). The primary effect of the target-scale hydrodynamics is the curvature of the shock and interface. As noted previously, the curvature in the simulation is greater than in the experiment and, as a result, the transmitted shock speed on axis is too low at late times. There is a Mach stem apparent in the simulations near the edge of the shock tube, but its presence in the experiment cannot be confirmed or refuted based on the radiographs available. The resolution in the full-target simulation (30 ppw) is only half that typically used in the half-wavelength simulation. Nevertheless, the gross features of the experiment are apparent in the simulation. In particular, the simulation reproduces the release waves originating from the shock tube walls. These waves do not reach the target axis until about $25 \mathrm{~ns}$, and so cannot affect the on-axis interface structure until very late times.

The amplitude history produced by a CALE halfwavelength simulation agrees (to within the experimental resolution of about $10 \mu \mathrm{m}$ ) with amplitude data extracted from the radiographs shown in Fig. 10 (see Fig. 11). The reason that this is true for a single simulation despite the experimental variation in drive energy is that the incident shock velocity scales as the one-third power of the drive intensity. For the data points shown, the experimental resolution is between $7 \%$ and $15 \%$ of the interpenetration width. Thus, the apparent agreement in amplitude does not conflict 
(a)

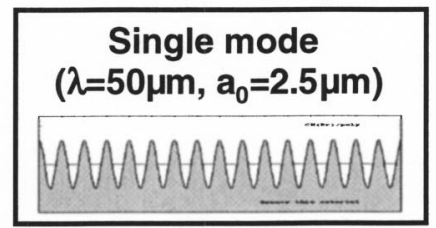

(b)
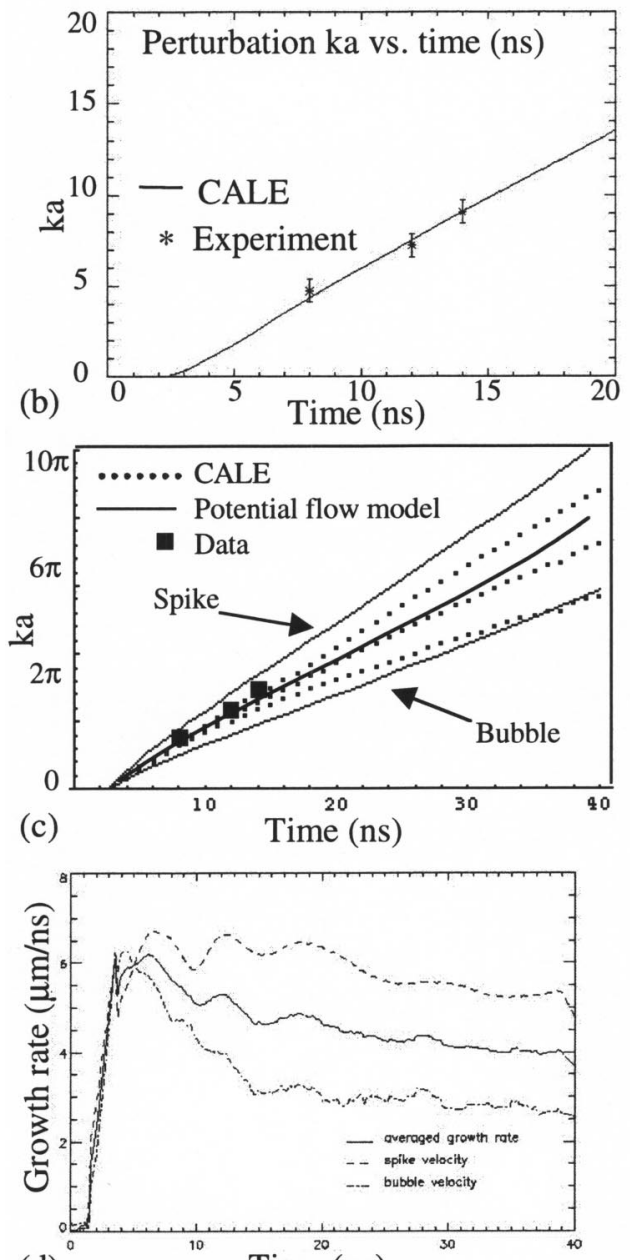

(d)

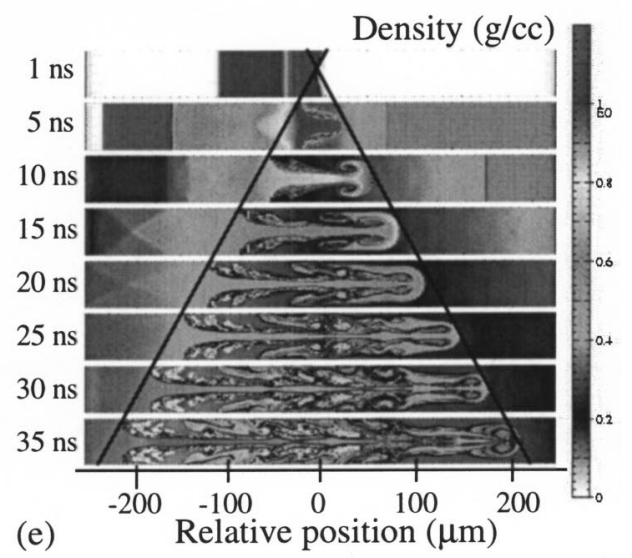

FIG. 11. (a) Single-mode perturbation. (b) Comparison of the simulated amplitude history with the experimental data show good agreement. (c) Comparison of the data with the experiment and Goncharov's potential flow model with decompression. (d) Spike, bubble, and averaged amplitude growth rates. (e) Density plots show the development of highly nonlinear spike and bubble structure. The simulation in (b) and (e) use LEOS for the plastic and EOP for the foam and a $3.5 \mathrm{~kJ}$ drive, while (c) and (d) use EOP for both materials and a $5 \mathrm{~kJ}$ drive. with the obvious over-prediction of the transmitted shock displacement at late times. The latter is of order $10 \%$ after 10 ns.

After shock transmission, the instability developing at the interface evolves almost immediately through the linear regime (in about $1 \mathrm{~ns}$ ), becomes nonlinear, and continues well into the deep nonlinear regime. The degree of nonlinearity attained in the simulation is apparent in the amplitude plot [Fig. 11(c)], which shows that $k a$ reaches a value of about 29 at $40 \mathrm{~ns}$, as well as in the series of density plots shown in Fig. 11(e). An asymmetric spike and bubble structure, typical of the nonlinear RT and RM instabilities, has developed by $5 \mathrm{~ns}$ ( $2.5 \mathrm{~ns}$ after shock transmission). At later times, the spike becomes a long thin structure and develops prominent rollups at its tip.

In the nonlinear phase, the amplitude growth is approximately linear in time despite the fact that the deceleration at the interface, and consequently the RT growth rate, is approaching zero (see Fig. 4). This behavior can be attributed to the decompression of the target in the expansion fan behind the shock front. Most nonlinear RT models are incompressible, and therefore do include perturbation growth resulting from target decompression. However, Goncharov has developed a potential flow model with a time-dependent density profile. ${ }^{31}$ The model captures the general behavior of the perturbation, but typically overpredicts the spike growth and underpredicts the bubble growth [see Fig. 11(c)]. In order to compare the simulation results with models of the RT instability that do not include material expansion, we must first subtract the expansion contribution from the spike and bubble amplitude histories. Separate spike and bubble amplitudes are obtained by simply subtracting the interface position, predicted by a 1D simulation, from the spike and bubble positions. The time-dependent fluid velocities at the spike and bubble positions are extracted from the same 1D simulation, and are interpreted as the spike and bubble expansion velocity histories. Integration of these functions yields the expansion amplitude histories, which are then subtracted from the actual amplitude histories to obtain the expansion-corrected RT spike and bubble amplitudes. Even after subtraction of the decompression effect, the spike and bubble amplitudes grow up to values of $k a \approx 13$ and $k a \approx 9$, respectively. In Fig. 12, the decompression-corrected amplitude curves are compared to the prediction of the buoyancydrag model of Oron et al. ${ }^{32}$ The model, which follows earlier work by Hanson et al. ${ }^{33}$ and Dimonte, ${ }^{34}$ predicts that $2 \mathrm{D}$ bubbles "rise" with a velocity determined by

$$
\left(\rho_{b}+2 \rho_{s}\right) \frac{d u_{b}(t)}{d t}=\left(\rho_{s}-\rho_{b}\right) g(t)-\frac{6 \pi}{\lambda} \rho_{s} u_{b}^{2}
$$

The equation for the spike velocity is obtained by simply interchanging spike and bubble densities. For 3D spikes and bubbles, the numerical coefficients in Eq. (2) change from two to one and from six to two. The initial spanwise symmetry might eventually be broken in the experiments, but the simulations of course remain 2D. Consequently, the 2D coefficients remain the valid choice for comparison with the simulation at all times. As noted by Dimonte, ${ }^{34}$ such models 


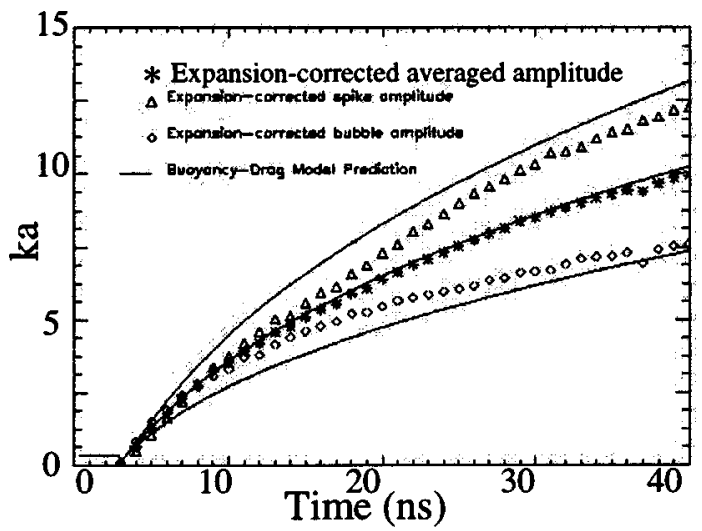

FIG. 12. Comparison of the simulation results with the buoyancy-drag model of Oron et al. [Phys. Plasmas 8, 2883 (2001)]. The simulation is driven by a $3.5 \mathrm{~kJ}$ pulse and uses EOP for all materials.

tend to overestimate the spike-bubble asymmetry. This results from their application at early times when the perturbation has not yet reached its asymptotic state. In our case, it could also result from our approximate treatment of the RM effects, which dominate the instability growth early on. In any event, the averaged amplitude is nevertheless well predicted by the model throughout the simulation and well into the deep nonlinear regime. In addition, Eq. (2) gives a qualitatively correct description of the spike and bubble behavior. At late times, the spike and bubble velocities decay along with the driving interface acceleration, with the asymptotic spike amplitude significantly higher than that of the bubble.

\section{RM contribution}

In applying the buoyancy-drag model to the simulation results, the effect of the RM instability was included by initializing the spike and bubble velocities with the MeyerBlewett velocity. ${ }^{35}$ The Meyer-Blewett velocity is essentially Richtmyer's original impulsive model, ${ }^{25}$ given by

$$
v_{\mathrm{MB}}=k a^{*} A^{*} u_{i},
$$

adapted to the case of a shock passing from a heavier to a lighter fluid. This is done by simply replacing the post-shock perturbation amplitude $a^{*}$ in the Richtmyer formula with the average of the pre- and post-shock amplitudes. In either case, $A^{*}$ is the post-shock Atwood number and $u_{i}$ is the velocity increase of the interface upon shock transmission. Evaluation of Eq. (3) for the simulation gives $v_{\mathrm{MB}}=6 \mu \mathrm{m} / \mathrm{ns}$. Interestingly, this is precisely the early-time peak amplitude growth rate seen in Fig. 11(d), suggesting that the perturbation growth is dominated by the RM instability during the first couple of nanoseconds.

In order to make some estimate of the RM contribution at all times, we ran a simulation in which a modified target was driven by a constant velocity $(41.5 \mu \mathrm{m} / \mathrm{ns})$ piston. The piston velocity and pusher thickness were chosen such that the resulting long, steady shock would accelerate the interface up to the same velocity as that provided by the blast wave, and then maintain that same velocity without deceleration [see Fig. 13(a)]. The amplitude and velocity histories [Figs. 13(b) and 13(c)] display classical RM behavior. The
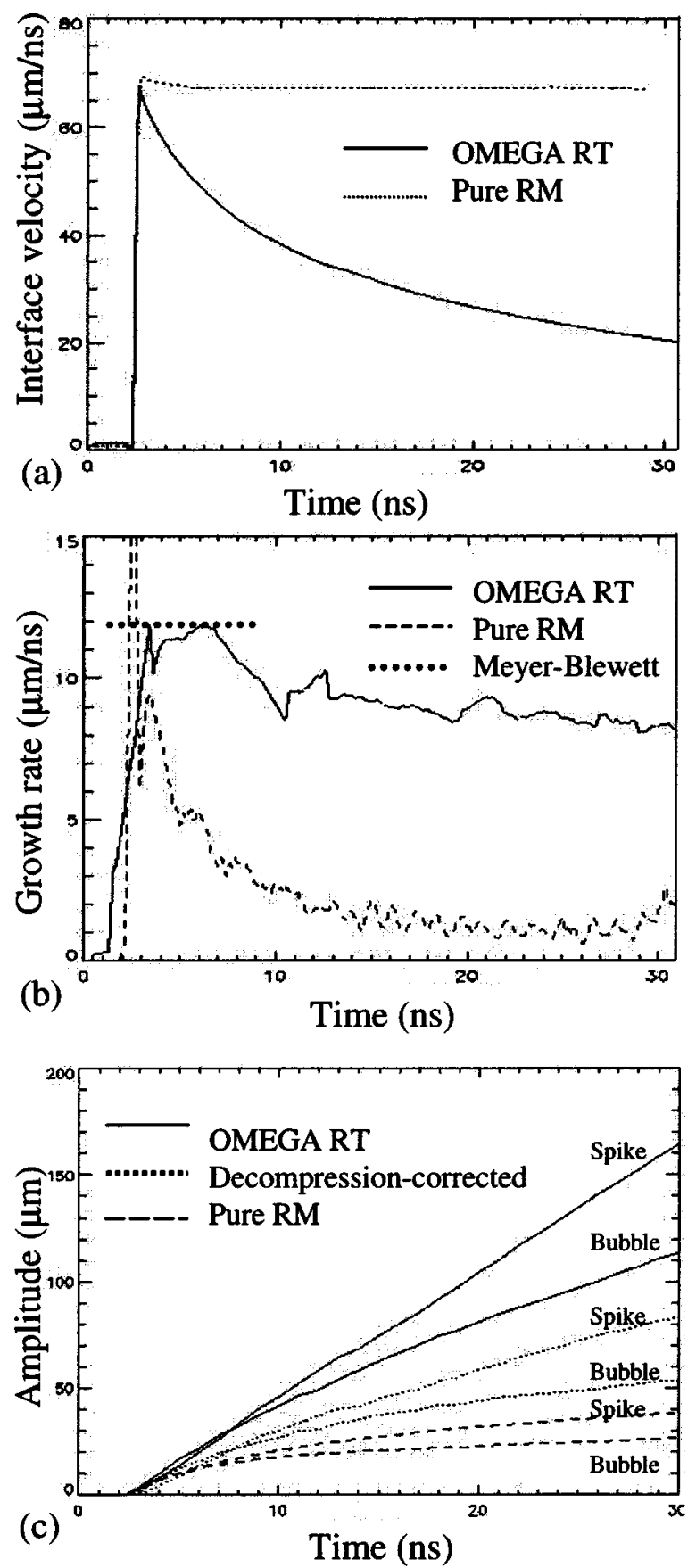

FIG. 13. Estimation of contribution from RM instability and decompression. (a) Interface velocities resulting from OMEGA RT (3.5 kJ) and pure RM (24 ns $41.5 \mu \mathrm{m} / \mathrm{ns}$ ) drives. Pure RM (b) amplitude growth rate shows classic RM behavior. The peak amplitude growth rate is well predicted by the Meyer-Blewett velocity of $6 \mu \mathrm{m} / \mathrm{ns}$. RT dominates over RM after a few ns. (c) Spike and bubble amplitude histories from the simulation of the experiment, expansion corrected RT+RM simulation, and the pure RM simulation. Decompression accounts for about $50 \%$ of the total perturbation growth at late times, and RM accounts for up to $50 \%$ of the expansioncorrected perturbation growth.

amplitude growth rate quickly climbs to a value that is reasonably well predicted by the Meyer-Blewett formula, and then decays while undergoing oscillations. Comparison with the amplitude growth rate observed in the RT simulation shows that the RM contribution, while initially responsible for virtually all the perturbation growth, falls to half the total 
after about $3 \mathrm{~ns}$ and becomes relatively insignificant shortly thereafter. This is not because the RT amplitude growth rate, which is proportional to perturbation amplitude in the linear regime, has grown much larger than the Meyer-Blewett velocity. Indeed, the total amplitude growth rate never exceeds the Meyer-Blewett velocity. Rather, it results from the relatively quick decay of the RM driving mechanism. At $30 \mathrm{ns,}$ the spike and bubble amplitudes in the pure RM simulation are roughly $50 \%$ of their decompression-corrected counterparts in the actual experiment. Despite its relatively low contribution to the growth rate during most of the experiment, therefore, the RM instability may contribute up to about half of the decompression-corrected perturbation growth [see Fig. 13(c)]. An estimate of the RM contribution obtained in this way should be considered as an upper bound, since the effects of the two instabilities do not necessarily add up linearly. Such uncertainty does not exist in the consideration of the combined contribution of the RT and RM instabilities relative to the total growth including decompression. The RT + RM contribution begins at $100 \%$, falls to $50 \%$ at about $20 \mathrm{~ns}$, is between $45 \%$ and $50 \%$ at $30 \mathrm{~ns}$, and continues to diminish at still later times.

\section{Equation of state}

The simulation results are sensitive to the choice of EOS model specified in CALE. This sensitivity was investigated through a series of simulations involving different EOS combinations. The study included the EOP and LEOS tables as well as perfect gas models with a range of choices for the adiabatic indices $\gamma$ of the plastic pusher and foam payload. Relevant portions of the EOP and LEOS shock Hugoniot curves for the plastic pusher and foam payload are shown in Fig. 14. In regions of the curves accessed by the incident and transmitted shocks, EOP predicts greater material compressibility than does LEOS. This is particularly true for the foam, where the resulting limiting compression factor with EOP is about $30 \%$ greater than with LEOS.

The choice of EOS affects the interface and shock velocities, perturbation amplitude, and the spike and bubble shape (see Figs. 15 and 16). However, the experimental resolution is insufficient to distinguish between EOS models based on their predictions of the spike and bubble shape. The perturbation amplitude is reasonably well predicted by several of the models, which on average vary from the data by about $10 \%$, but the combination of LEOS for the plastic and EOP for the foam does the best job. The growth rate decreases as the foam compressibility increases, and the data are inconsistent with foam modeled as an ideal gas with adiabatic index less than 1.4. The transmitted shock speed is over-predicted by half-wavelength simulations using both the EOP and LEOS tables. As noted previously, this is partially due to lateral expansion behind the curved shock front that is not present in the half-wavelength simulations. However, this effect is small because of the front's large radius of curvature, and in any event does not explain the EOS model dependence seen in simulations. The LEOS tables in particular appear to greatly overstate the foam stiffness. This tendency has been noted in simulations of OMEGA RM

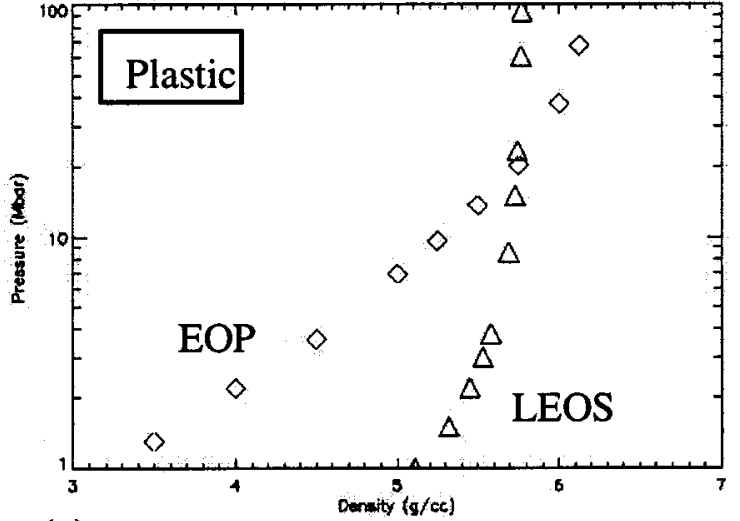

(a)

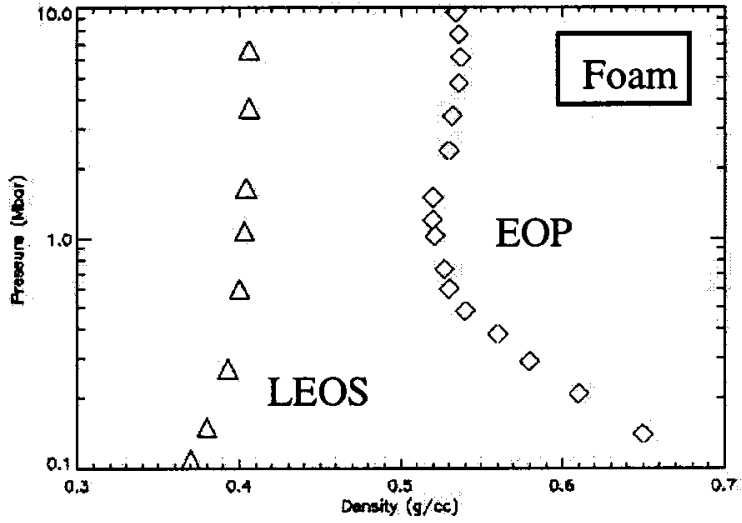

(b)

FIG. 14. Tabular EOS shock Hugoniot curves in relevant ranges for (a) brominated polystyrene (used for plastic pusher) with $\rho_{0}=1.42 \mathrm{~g} / \mathrm{cc}, T_{0}$ $=25 \mathrm{meV}$ and (b) polystyrene (used for foam payload) with $\rho_{0}=0.1 \mathrm{~g} / \mathrm{cc}$, $T_{0}=25 \mathrm{meV}$. In both cases, EOP is more compressible than LEOS at very high pressures. The difference is particularly pronounced for the foam.

experiments, ${ }^{27}$ but the discrepancy here is greater than that seen previously.

It is possible to match one or another of the key observable parameters by resorting to a perfect gas model and adjusting the adiabatic indices, but this is usually at the expense of the agreement of some other parameter. For example, we can reduce the transmitted shock speed by reducing $\gamma_{\text {foam }}$, thereby increasing the compressibility of the foam. But the resulting amplitude is soon reduced clearly below that seen in the experiment. At the same time, the interface speed becomes too high. Clearly (and not surprisingly), the materials in question do not behave as ideal gases at the energy densities accessed in these experiments and simulations.

Considering together the dependence of the perturbation amplitude history and the interface and shock trajectories found in the single-mode simulations, it seems that the target materials are best represented by LEOS tables for the plastic pusher and EOP tables for the carbon foam payload. Similar comparisons made with simulations of the multimode experiments lead to the same conclusion. Consequently, the multimode. simulations presented in the next sections all use this same combination.

\section{E. Multimode perturbations}

Having demonstrated the ability to accurately simulate and model the unstable evolution of single-mode driven by a 


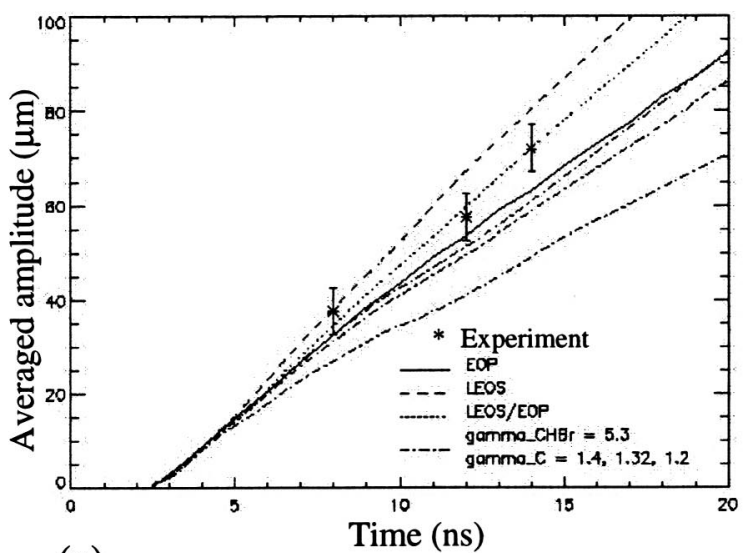

(a)

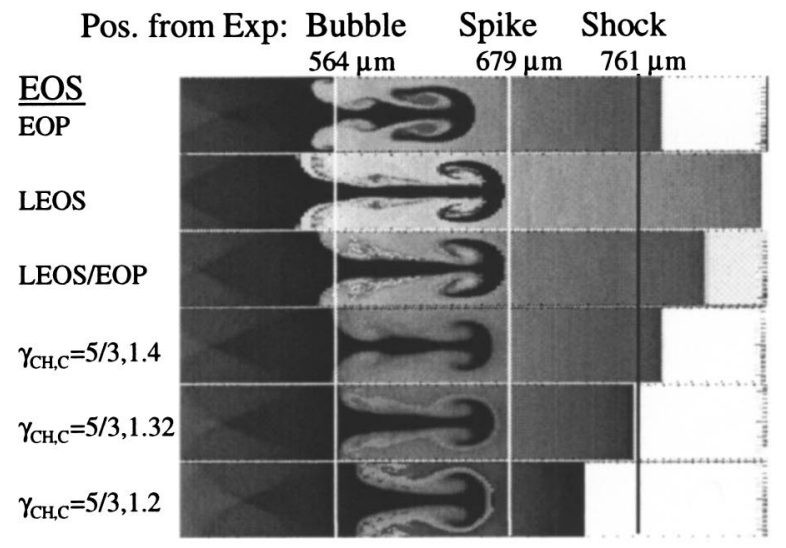

(b)

FIG. 15. EOS sensitivity. (a) Amplitude histories from CALE simulations run with several different EOS combinations. For perfect gas models, the amplitude increases with decreasing foam compressibility. (b) Numerical radiographs at $12 \mathrm{~ns}$. The combination of LEOS for the plastic and EOP for the foam provides the best agreement with the data. Simulations run with either LEOS or EOP overpredict the transmitted shock speed.

strong blast wave, we now turn to the evolution of multimode interfaces. Ultimately, we wish to understand the growth of highly turbulent mixing layers present within supernovae. Rather than beginning with a broad spectrum, we first consider interfaces that are initially two and eight mode in order to investigate the approach and transition to turbulence. This will lay the groundwork for later studies with many initial modes on a wider range of scales.

\section{Two mode}

The two-mode perturbation [see Fig. 17(a)] is characterized by a sum of two sinusoidal components (in phase) with wavelengths of 40 and $60 \mu \mathrm{m}$ and amplitudes of 1.25 and 1.5 $\mu \mathrm{m}$, respectively. This results in an initial perturbation width that is very near to the single-mode target value of $5 \mu \mathrm{m}$.

As is apparent from the simulated radiograph in Fig. 17(b), the interface structure seen in the two-mode CALE simulation at $13 \mathrm{~ns}$ is in strikingly good agreement with the data. The simulation interpenetration width at this time was $122 \mu \mathrm{m}$, in agreement with the measured value $130 \pm 10 \mu \mathrm{m}$. The code prediction at $26 \mathrm{~ns}$ [see Fig. 17(c)] appears to be fairly good as well, but is complicated by the degradation in

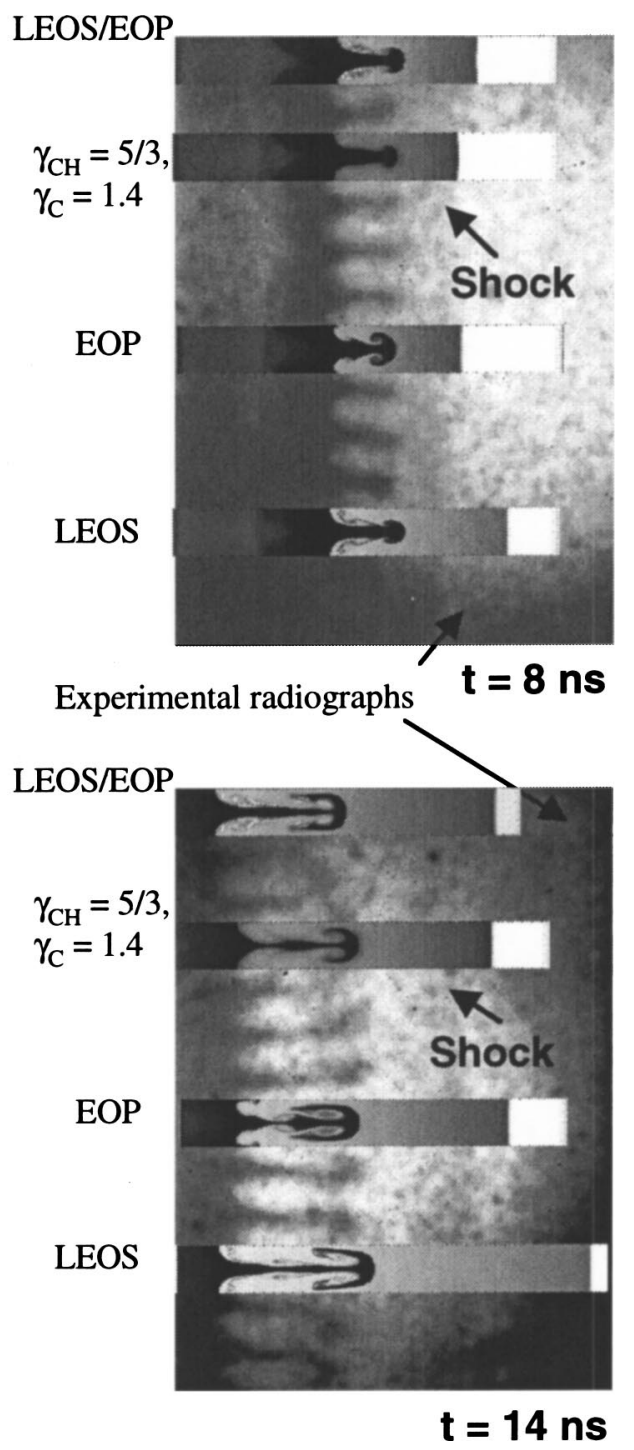

FIG. 16. EOS. Choosing a good EOS model is important for matching the experiment. LEOS for the plastic with EOP for the foam is best at getting the perturbation amplitude. Perfect gas with $\gamma_{\text {plastic }}=5 / 3, \gamma_{\text {foam }}=1.4$ is best at getting the shock position relative to the interface, while perfect gas with $\gamma_{\text {plastic }}=5 / 3, \gamma_{\text {foam }}=1.32$ is best at getting the absolute shock position. Differences in spike and bubble shape are apparent in the simulations, but the data are not good enough to distinguish between the models on this basis.

contrast by that time. It is quite possible that $3 \mathrm{D}$ effects have begun to appear in the experiment, marking close approach to the transition to turbulence and the limitations of the $2 \mathrm{D}$ simulation. The simulated mix width, $307 \mu \mathrm{m}$, is slightly larger than the observed value of $294 \pm 10 \mu \mathrm{m}$. It is clear from both images that, as in the single-mode simulations, the code overpredicts the transmitted shock speed.

The qualitative success of the buoyancy-drag model in predicting the single-mode spike and bubble growth is not apparent when the model is applied to the two-mode interface [see Fig. 17(d)]. In applying the model to the spike growth, the time-dependent transverse width of the outlying spike was used in place of the wavelength in Eq. (2). In the case of the bubble growth, this method would lead to a gross underprediction of the observed growth at all times. Instead, the model is initialized at the largest wavelength in the prob- 


\section{(a) Two mode
$\left(\lambda=40 \& 60 \mu \mathrm{m}, a_{0}=2.5 \mu \mathrm{m}\right)$}
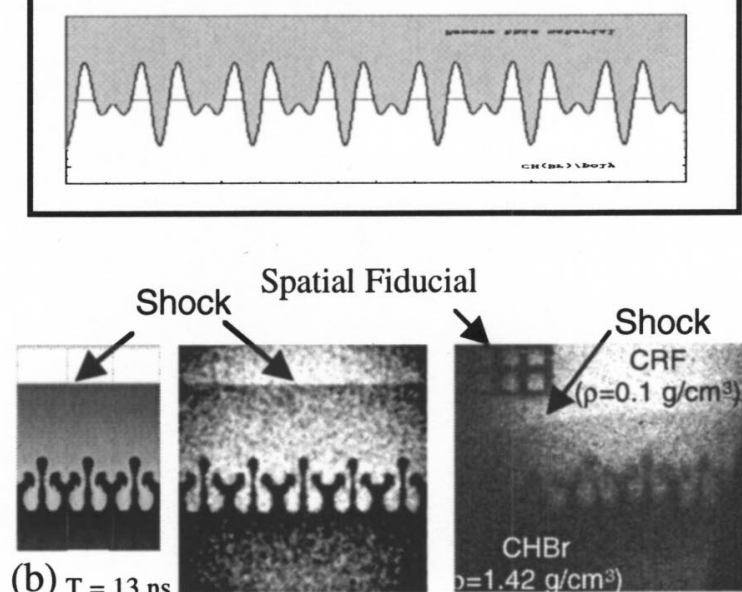

(b) $\mathrm{T}=13 \mathrm{~ns}$ $\begin{array}{ll}\text { Numerical } & \text { Simulated Radiograph }= \\ \text { Radiograph } & \text { Numerical Radiograph }+\end{array}$ Radiograph
Experimental Noise
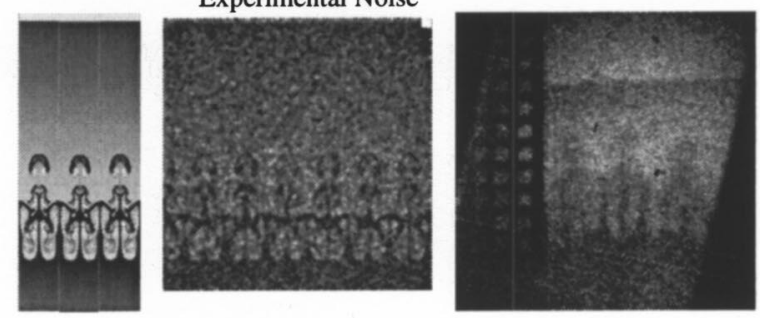

(c)

$$
\mathrm{T}=26 \mathrm{~ns}
$$

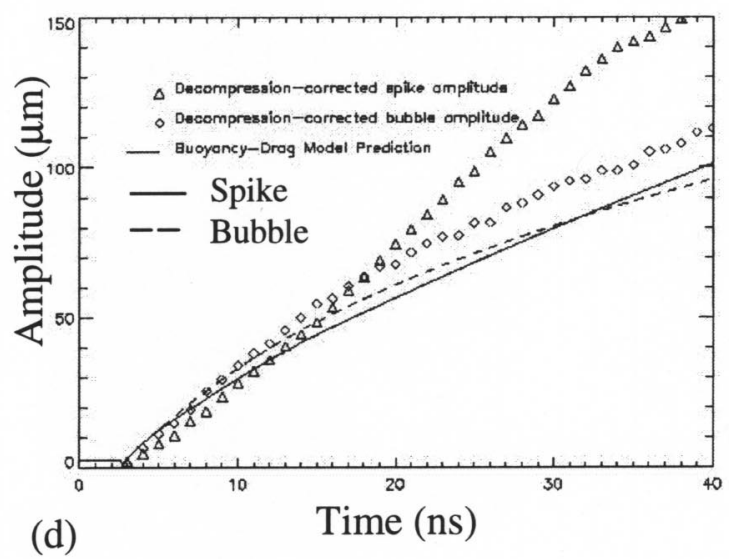

FIG. 17. Two-mode perturbation simulation and data. (a) Imposed interface perturbation. (b) Again, the spike and bubble size and structure look good, but the transmitted shock speed is clearly too high. (c) Comparison of decompression-corrected simulation results with the buoyancy-drag-model prediction shows only early-time agreement.

lem. After the first (and only) bubble merger event occurs at about $6 \mathrm{~ns}$, this wavelength is replaced by the largest transverse scale possible in the simulation-twice the $60 \mu \mathrm{m}$ box size. The resulting model-predicted behavior of the spike and bubble is similar to that observed in the single-mode case. That is, the spike and bubble velocities begin large and decay smoothly in time. In this case, however, the spike and bubble are predicted to grow nearly symmetrically. In the simulation, the bubble growth is consistent with the model description. The spike velocity, on the other hand, abruptly increases at about $15 \mathrm{~ns}$ and subsequently undergoes a period of growth that is nearly linear in time. This fact, coupled with the agreement between the simulation and experiment, suggests the significant influence of compressibility or some other phenomenon outside the model's range of validity.

\section{Eight mode}

The initial perturbation in the eight-mode targets [see Fig. 18(a)] is defined by the sum

$$
a(r)=\sum_{i=1}^{8} a_{i} \cos \left(\frac{2 \pi r}{\lambda_{i}}\right),
$$

where the wavelength vector is given by $\lambda_{i}=180 \mu \mathrm{m} / i$ and the components of the amplitude vector range in absolute value between 0.4 and $0.7 \mu \mathrm{m}$. The initial perturbation width is $4.9 \mu \mathrm{m}$-again very close to the single-mode case.

As in the two-mode case, the simulation results at $13 \mathrm{~ns}$ reproduce most of the features seen in the experiment, so that the simulation is in good qualitative agreement with the data on a range of scales from the largest down to the experimental resolution [see Fig. 18(b)]. The simulation interpenetration width at this time is $134 \mu \mathrm{m}$, somewhat larger than the observed value of $120 \pm 10 \mu \mathrm{m}$. Comparison with the $26 \mathrm{~ns}$ data is even more problematic than was the case with the two-mode perturbation [Fig. 18(c)]. The numerical radiograph displays a dominant $180 \mu \mathrm{m}$ mode on top of which is overlaid a feathery smaller-scale spike and bubble structure. The same dominant mode is apparent in the data, but the feathery structure is not. In fact, nothing at all is seen to protrude clearly out beyond the dominant mode. This could simply result from a loss of contrast associated with decreasing density and the appearance of 3D effects. But it could also indicate that the smaller-scale structure has already transitioned to turbulent flow following the generation of initially laminar 3D structure. Considering both the Reynolds number of the flow and the time over which it is maintained in these experiments, the evolving interface could in fact undergo a turbulent mixing transition at as early as about 17 ns. ${ }^{23}$ The experimental images of the two-mode interface also show a significant loss of contrast between 13 and $26 \mathrm{~ns}$, though not so drastic as in the eight-mode case. While these data are suggestive, more experiments will be necessary before a firm conclusion about transition can be made. These experiments may require greater resolution and contrast between spike and bubble material, and should eventually explore the dependence on initial dimensionality and modal spectrum.

Our application of the buoyancy-drag model to the eightmode case is shown in Fig. 18(d). In contrast with the twomode case, it is now the bubble front that exhibits anomalous behavior. The bubble's amplitude and velocity are both considerably greater than those of the spike after about $20 \mathrm{~ns}$. Despite our efforts to account for bubble expansion and merger, we were again unable to show even qualitative agreement of the model with the simulation. 

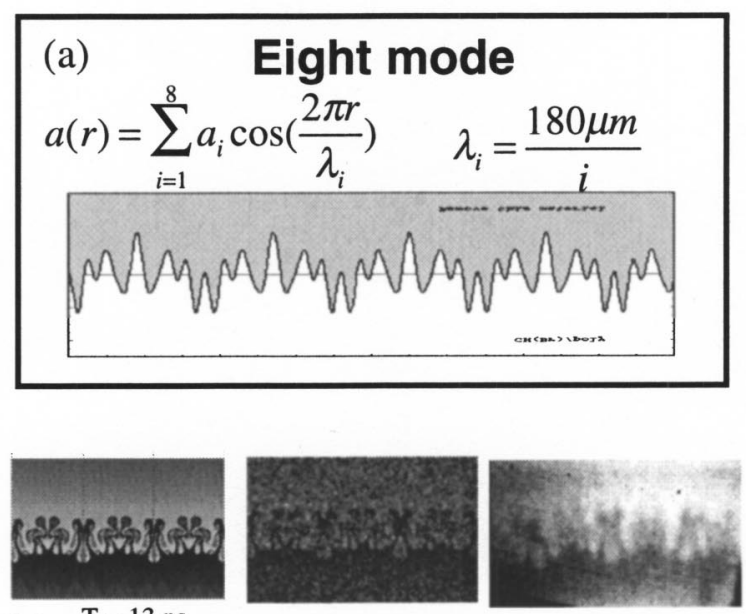

(b) $\mathrm{T}=13 \mathrm{~ns}$

Simulated Radiograph $=$ Numerical Numerical Radiograph + Radiograph Experimental Noise Experimental Radiograph
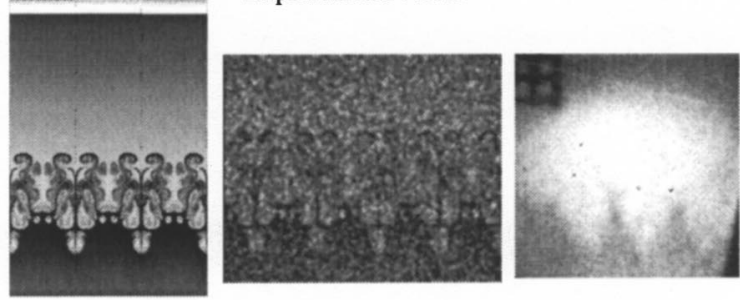

(c)

$$
\mathrm{T}=26 \mathrm{~ns}
$$

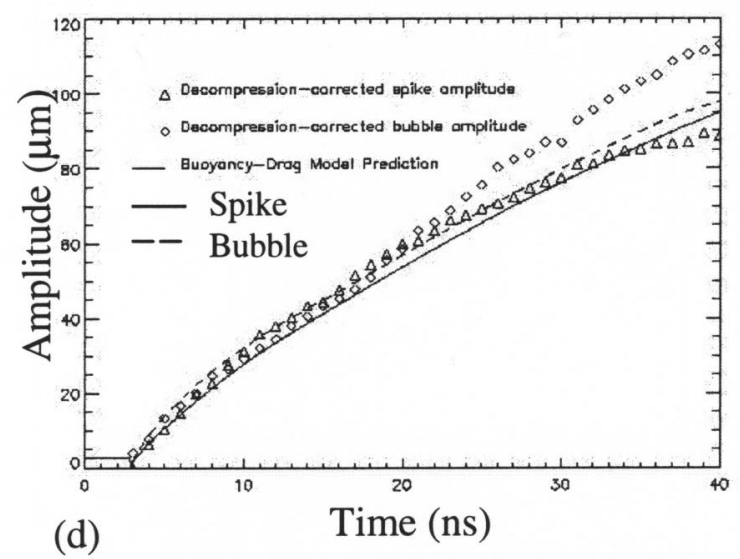

FIG. 18. Eight-mode perturbation simulation and data. (a) Imposed interface perturbation. (b) CALE results are qualitatively in good agreement with the eight-mode data. (c) Comparison of decompression-corrected simulation results with the buoyancy-drag-model prediction shows only early-time agreement.

\section{CONCLUSIONS AND FUTURE DIRECTIONS}

We have presented the results of 2D CALE simulations of blast-wave-driven hydro experiments performed at OMEGA. Simulations of single-, two-, and eight-mode targets are in reasonable agreement with experiments on a range of scales from large down to the experimental resolution. In addition, the single mode evolution is well predicted by a buoyancy-drag model when the effects of target decompression are first subtracted away. We were unable, however, to use the model to correctly describe the evolution of the multimode spike and bubble fronts even when bubble expan- sion and merger were accounted for. The partial success of the model will be further discussed and explained in a later paper.

Eulerian calculations appear to better reproduce the spike and bubble shapes than do the ALE runs, but the comparison is limited by the current resolution and the effect on the perturbation amplitude is negligible. The dependence of the instability evolution on the laser energy and pulse shape, $\mathrm{x}$-ray preheat, and the EOS model have also been considered. The first three factors affect the amplitude at or below the $5 \%$ level, but the choice of EOS, from commonly used tables, can significantly affect both the amplitude growth rate (at about the 10\% level) and the interface structure. All of these factors must be given careful attention in detailsensitive simulations of experiments.

Analysis of the simulation data suggests that the RT and RM instabilities contribute roughly equally to the decompression-corrected perturbation growth, with RM dominant in the first few ns and RT dominant at later times. Together, the two instabilities account for about $50 \%$ of the total growth, with the remaining half resulting from target decompression. Consequently, all three effects must be considered in order to accurately describe the perturbation growth.

Because of limited computational resources, the simulation Reynolds number is two orders of magnitude smaller than in the experiment (currently $\mathrm{Re}^{\mathrm{CALE}} \sim 1200$ when $\operatorname{Re}^{\text {expt }} \sim 10^{5}$ ). The hypothesized critical Re for the mixing transition lies in between these two values. The inherent 2D nature of the calculations provides an even more important limitation on their ability to reproduce all aspects of the latetime instability evolution. Consequently, the simulations cannot reproduce the smallest-scale features present in the experiment. If we consider structures computationally unresolved if their spatial scale is less than 10 grid cells, then all unresolved scales are below the current experimental resolution. Therefore, the limited range of scales present in the simulations is important only when there is significant coupling between the large and small scales. Good agreement between data and simulations indicate that this is not the case until sometime after $14 \mathrm{~ns}$.

Having demonstrated the ability to accurately simulate the late nonlinear stages of the instability evolution for both single-mode and multimode perturbations, we can now with some degree of confidence proceed to model data taken at later times, as the instability approaches and, hopefully, passes through the transition to turbulence. We can also move to 3D calculations to investigate when and how the 2D symmetry of the experiments is broken. Since the simulations cannot reach the high Reynolds numbers present in the experiments, it will be interesting to observe the code predictions at the transition time as predicted by theory and observed in experiments. In fact, sudden deviation of simulation from data, after a period of good agreement, may be the best indication that transition has taken place. Continuing improvement in experimental resolution will of course also be helpful. With these tools, we intend to study the dependence of the time to transition on the initial modal spectrum. A key question is how and when will the initial conditions be 
"forgotten" and the instability proceed into a self-similar regime? Finally, what are the absolute limits of current computer codes and hardware resources in accurately modeling complex high-Reynolds number flows. These questions must be addressed if a real understanding of the nonlinear hydrodynamics present in core-collapse supernovae is to be attained.

\section{ACKNOWLEDGMENTS}

The authors acknowledge useful contributions from P. A. Keiter, K. E. Korreck, M. Blackburn, M. Grosskopf, B. A. Remington, R. J. Wallace, and H. Louis.

This work was performed under the auspices of the U.S. Department of Energy by the University of California, Lawrence Livermore National Laboratory under Contract No. W-7405-Eng-48. Work at the University of Michigan was supported by the U.S. Department of Energy under Grants Nos. DE-FG03-99DP00284, DE-FG03-00SF22021, and other grants and contracts.

${ }^{1}$ J. W. S. Rayleigh, Scientific Papers (University Press, Cambridge, 1899).

${ }^{2}$ G. I. Taylor, Proc. R. Soc. London, Ser. A 201, 192 (1950).

${ }^{3}$ S. Chandrasekhar, Hydrodynamic and Hydromagnetic Stability (Dover, New York, 1981).

${ }^{4}$ J. D. Lindl, Inertial Confinement Fusion: The Quest for Ignition and HighEnergy Gain (Springer-Verlag, New York, 1998).

${ }^{5}$ D. Shvarts, O. Sadot, D. Oron, A. Rikanati, U. Alon, and G. Ben-Dor, in Handbook of Shock Waves, edited by G. Ben-Dor, O. Igra, and T. Elperin (Academic, London, 2001), Vol. 2, Chap. 14, pp. 489-543.

${ }^{6}$ H. J. Kull, Phys. Rep. 206, 197 (1991).

${ }^{7}$ M. N. Rosenbluth and C. L. Longmire, Ann. Phys. (N.Y.) 26, 2227 (1957).

${ }^{8}$ R. Chevalier, Astrophys. J. 207, 872 (1976).

${ }^{9}$ Ya. B. Zel'dovich and Yu. P. Raizer, Physics of Shock Waves and HighTemperature Hydrodynamic Phenomema (Dover, New York, 2002).

${ }^{10}$ National Research Council of the National Academies, Frontiers in High Energy Density Physics (The National Academies Press, Washington DC, 2002).

${ }^{11}$ W. D. Arnett, J. N. Bachall, R. P. Kirshner, and S. E. Woosley, Annu. Rev. Astron. Astrophys. 27, 629 (1989).
${ }^{12}$ W. Hillebrandt and P. Hoflich, Rep. Prog. Phys. 52, 1421 (1989).

${ }^{13}$ D. Ryutov, R. P. Drake, J. Kane, E. Liang, B. A. Remington, and W. M. Wood-Vasey, Astrophys. J. 518, 821 (1999).

${ }^{14}$ D. Arnett, B. Fryxell, and E. Muller, Astrophys. J. Lett. 341, L63 (1989); E. Muller, B. Fryxell, and D. Arnett, Astron. Astrophys. 251, 505 (1992).

${ }^{15}$ B. A. Remington, J. Kane, R. P. Drake et al., Phys. Plasmas 4, 1994 (1997).

${ }^{16}$ J. Hecht, U. Alon, and D. Shvarts, Phys. Fluids 6, 4019 (1994).

${ }^{17}$ U. Alon, J. Hecht, D. Ofer, and D. Shvarts, Phys. Rev. Lett. 74, 534 (1995).

${ }^{18}$ J. M. Soures, R. L. McCrory, C. P. Verdon et al., Phys. Plasmas 3, 2108 (1996).

${ }^{19}$ J. Kane, D. Arnett, B. A. Remington, S. G. Glendinning, J. Castor, R. Wallace, A. Rubenchik, and B. A. Fryxell, Astrophys. J. Lett. 478, L75 (1997).

${ }^{20}$ B. A. Remington, R. P. Drake, H. Takabe, and D. Arnett, Phys. Plasmas 7, 1641 (2000)

${ }^{21}$ R. P. Drake, J. J. Carroll, K. Eastbrook, S. G. Glendinning, B. A. Remington, and R. McCray, Astrophys. J. Lett. 500, L157 (1998).

${ }^{22}$ P. E. Dimotakis, J. Fluid Mech. 409, 69 (2000).

${ }^{23}$ H. F. Robey, Y. Zhou, A. C. Buckingham, P. Keiter, B. A. Remington, and R. P. Drake, Phys. Plasmas 10, 614 (2003).

${ }^{24}$ R. T. Barton, Numerical Astrophysics (Jones and Bartlett, Boston, 1985).

${ }^{25}$ R. D. Richtmyer, Commun. Pure Appl. Math. 13, 297 (1960).

${ }^{26}$ E. E. Meshkov, Izv. Akad. Nauk SSSR, Mekh. Zhidk. Gaza 4, 151 (1969).

${ }^{27}$ S. G. Glendinning, J. Bolstad, D. G. Braun et al., Phys. Plasmas 10, 1931 (2003).

${ }^{28}$ R. M. More, K. H. Warren, D. A. Young, and G. B. Zimmerman, Phys. Fluids 31, 3059 (1988).

${ }^{29}$ S. V. Weber, B. A. Remington, S. W. Haan, B. G. Wilson, and J. K. Nash, Phys. Plasmas 1, 3652 (1994).

${ }^{30}$ G. B. Zimmerman and W. L. Kruer, Comments Plasma Phys. Controlled Fusion 2, 51 (1975).

${ }^{31}$ V. N. Goncharov, P. McKenty, S. Shupsky, R. Betti, R. L. McCrory, and C. Cherfils-Clerouin, Phys. Plasmas 7, 5118 (2000).

${ }^{32}$ D. Oron, L. Arazi, D. Kartoon, A. Rikanati, U. Alon, and D. Shvarts, Phys. Plasmas 8, 2883 (2001).

${ }^{33}$ J. C. V. Hanson, P. A. Rosen, T. J. Goldsack, K. Oades, P. Fieldhouse, N. Cowperthwaite, D. L. Youngs, N. Mawhinney, and A. J. Baxter, Laser Part. Beams 8, 51 (1990).

${ }^{34}$ G. Dimonte, Phys. Plasmas 7, 2255 (2000).

${ }^{35}$ K. A. Meyer and P. J. Blewett, Phys. Fluids 15, 753 (1972). 